TRANSACTIONS OF THE

AMERICAN MATHEMATICAL SOCIETY

Volume 348, Number 6, June 1996

\title{
FINE STRUCTURE OF THE SPACE OF SPHERICAL MINIMAL IMMERSIONS
}

\author{
HILLEL GAUCHMAN AND GABOR TOTH
}

\begin{abstract}
The space of congruence classes of full spherical minimal immersions $f: S^{m} \rightarrow S^{n}$ of a given source dimension $m$ and algebraic degree $p$ is a compact convex body $\mathcal{M}_{m}^{p}$ in a representation space $\mathcal{F}_{m}^{p}$ of the special orthogonal group $S O(m+1)$. In Ann. of Math. 93 (1971), 43-62 DoCarmo and Wallach gave a lower bound for $\mathcal{F}_{m}^{p}$ and conjectured that the estimate was sharp. Toth resolved this "exact dimension conjecture" positively so that all irreducible components of $\mathcal{F}_{m}^{p}$ became known. The purpose of the present paper is to characterize each irreducible component $V$ of $\mathcal{F}_{m}^{p}$ in terms of the spherical minimal immersions represented by the slice $V \cap \mathcal{M}_{m}^{p}$. Using this geometric insight, the recent examples of DeTurck and Ziller are located within $\mathcal{M}_{m}^{p}$.
\end{abstract}

\section{INTRODUCTION}

Let $f: S_{\kappa}^{m} \rightarrow S_{V}$ be an isometric minimal immersion of the $m$-sphere $S_{\kappa}^{m}$, $m \geq 2$, of constant curvature $\kappa$ into the unit sphere $S_{V}$ of curvature 1 of a Euclidean vector space $V$. A result of Takahashi [7] asserts that $f$ exists iff $\kappa=\kappa_{p}=m / \lambda_{p}$, for some $p \geq 1$, where $\lambda_{p}=p(p+m-1)$ is the $p$-th eigenvalue of the Laplacian $\triangle^{S^{m}}$ on $S^{m}=S_{1}^{m}$ and, in this case, each component $\alpha \circ f, \alpha \in V^{*}$, of $f$ is an eigenfunction of the Laplacian $\triangle^{S_{\kappa}^{m}}$ with eigenvalue $m$ or, scaling the metric to curvature 1 , an eigenfunction of $\triangle^{S^{m}}$ with eigenvalue $\lambda_{p}$ that is, classically speaking, a spherical harmonic of order $p$ on $S^{m}$. The universal example is the standard minimal immersion $f_{m, p}: S^{m} \rightarrow S_{\mathcal{H}_{m}^{p}}$ that is defined by the requirement that, relative to a (scaled) $L^{2}$-orthonormal basis in the space of spherical harmonics $\mathcal{H}_{m}^{p}$ of order $p$ on $S^{m}$, the components of $f_{m, p}$ form an orthonormal basis. (Note that $p=2$ gives the classical Veronese maps.) Rigidity prevails for low ranges of the source dimension $m$ and the order $p$. In fact, Calabi [3] showed that, up to a congruence on the range, a full (= no zero component) isometric minimal immersion $f: S_{\kappa_{p}}^{2} \rightarrow S_{V}, p \geq 1$, is standard. Moreover, a general rigidity result of DoCarmoWallach [5, 11] specializes to saying that, for $p \leq 3$, again up to a congruence on the range, an isometric minimal immersion $f: S_{\kappa_{p}}^{m} \rightarrow S_{V}, m \geq 2$, is also standard.

For fixed $m \geq 2$ and $p \geq 1$, let $\mathcal{S}(m, p)$ denote the space of full isometric minimal immersions $f: S_{\kappa_{p}}^{m} \rightarrow S_{V}$, for various $V$. (Fullness geometrically means that the image of $f$ is not contained in any great hypersphere.) Two full isometric minimal immersions in $\mathcal{S}(m, p)$ are said to be congruent if they differ by an isometry between the ranges; this defines an equivalence relation on $\mathcal{S}(m, p)$, denoted by $\cong$. The

Received by the editors March 20, 1995.

1991 Mathematics Subject Classification. Primary 53C42.

(C)1996 American Mathematical Society 
DoCarmo-Wallach theory [5, 11] asserts that, for $m \geq 3$ and $p \geq 4$, the space of congruence classes $\mathcal{S}(m, p) / \cong\left(\right.$ of full isometric minimal immersions $f: S_{\kappa_{p}}^{m} \rightarrow S_{V}$ ) is nontrivial and can be parametrized by a compact convex body $\mathcal{M}_{m}^{p}$ lying in a representation space $\mathcal{F}_{m}^{p}$ of the special orthogonal group $S O(m+1)$. In fact, $\mathcal{F}_{m}^{p}$ is an $S O(m+1)$-submodule of the symmetric square $S^{2}\left(\mathcal{H}_{m}^{p}\right)$ of the space of spherical harmonics $\mathcal{H}_{m}^{p}$. The (complexification of the) symmetric square decomposes [5, 11] as

$$
S^{2}\left(\mathcal{H}_{m}^{p}\right)=\sum_{(u, v) \in \triangle_{0}^{p} ; u, v \text { even }} V_{m+1}^{(u, v, 0, \ldots, 0)}
$$

where $\triangle_{0}^{p} \subset \mathbf{R}^{2}$ is the closed convex triangle with vertices $(0,0),(p, p)$ and $(2 p, 0)$ and $V_{m+1}^{\left(u_{1}, \ldots, u_{d}\right)}, d=[|(m+1) / 2|]$, is the complex irreducible $S O(m+1)$-module with highest weight $\left(u_{1}, \ldots, u_{d}\right) \in(1 / 2) \mathbf{Z}^{d}$ relative to the standard maximal torus in $S O(m+1)$. In (1), $V_{m+1}^{(u, v, 0, \ldots, 0)}$ is not self-conjugate [2] iff $m=3$ and $v>0$ so that, in this case, it actually means $V_{4}^{(u, v)} \oplus V_{4}^{(u,-v)}$. Actually (1) follows from the recurrence relation $[5,11]$

$$
\mathcal{H}_{m}^{p} \otimes \mathcal{H}_{m}^{q}=\sum_{i=0}^{q} V_{m+1}^{(p+q-i, i, 0, \ldots, 0)} \oplus\left(\mathcal{H}_{m}^{p-1} \otimes \mathcal{H}_{m}^{q-1}\right), \quad p \geq q \geq 1, m \geq 2 .
$$

With this, we have [9]

$$
\mathcal{F}_{m}^{p}=\sum_{(u, v) \in \triangle_{2}^{p} ; u, v \text { even }} V_{m+1}^{(u, v, 0, \ldots, 0)}
$$

where the subtriangle $\triangle_{2}^{p} \subset \triangle_{0}^{p}$ has vertices $(4,4),(p, p)$ and $(2(p-2), 4)$. In fact, according to the main result of DoCarmo-Wallach $[5,11]$, the right hand side of (3) is contained in $\mathcal{F}_{m}^{p}$; they conjectured the stated equality in (3) that was proved (some 20 years later) in [9].

As noted above, scaling the metric on the source to curvature 1, aside from conformality, the components of a minimal immersion are spherical harmonics of a fixed order on the source. Disregarding conformality and retaining this latter condition, one arrives at the concept of an eigenmap. More precisely, $f: S^{m} \rightarrow S_{V}$ is said to be a $p$-eigenmap if each component $\alpha \circ f, \alpha \in V^{*}$, belongs to $\mathcal{H}_{m}^{p}$. A DoCarmo-Wallach type argument shows that in the nonrigid range $m \geq 3$ and $p \geq 2$, the set of congruence classes of full p-eigenmaps $f: S^{m} \rightarrow S_{V}$ can be parametrized by a compact convex body $\mathcal{L}_{m}^{p}$ in an $S O(m+1)$-submodule $\mathcal{E}_{m}^{p}$ of $S^{2}\left(\mathcal{H}_{m}^{p}\right)$. Its complexification decomposes [5, 11] (cf. also [8]) as

$$
\mathcal{E}_{m}^{p}=\sum_{(u, v) \in \triangle_{1}^{p} ; u, v \text { even }} V_{m+1}^{(u, v, 0, \ldots, 0)},
$$

where $\triangle_{1}^{p}$ has vertices $(2,2),(p, p)$ and $(2(p-1), 2)$ and is thereby nested between the previous triangles as

$$
\triangle_{0}^{p} \supset \triangle_{1}^{p} \supset \triangle_{2}^{p}
$$

Moreover, $\mathcal{M}_{m}^{p}$ is a linear slice of $\mathcal{L}_{m}^{p}$ cut out by $\mathcal{F}_{m}^{p}$.

A spherical harmonic of order $p$ on $S^{m}$ is nothing but the restriction (to $S^{m}$ ) of a harmonic $p$-homogeneous polynomial on $\mathbf{R}^{m+1}$. Thus, a $p$-eigenmap can be thought of as a harmonic $p$-homogeneous polynomial map $f: \mathbf{R}^{m+1} \rightarrow V$ that is spherical; that is, it maps the unit sphere $S^{m}$ to the unit sphere $S_{V}$. The set of congruence 
classes of full harmonic $p$-homogeneous polynomial maps can be parametrized by convex body $\mathcal{K}_{m}^{p}$ of $S^{2}\left(\mathcal{H}_{m}^{p}\right)$ and $\mathcal{L}_{m}^{p}$ is a linear slice of $\mathcal{K}_{m}^{p}$ cut out by $\mathcal{E}_{m}^{p}$.

Comparing (1), (3) and (4), we see that the chain of inclusions

$$
S^{2}\left(\mathcal{H}_{m}^{p}\right) \supset \mathcal{E}_{m}^{p} \supset \mathcal{F}_{m}^{p}
$$

corresponds to (restriction in (1) to) the nested triangles in (5), where each subtriangle is obtained by "slicing off" the base of the former. In addition, each $S O(m+1)$-module in (6) carries a geometric meaning, being the linear span of the parameter space of the congruence classes of full harmonic $p$-homogeneous polynomial maps, $p$-eigenmaps and, scaling the metric on the source to curvature 1 , conformal minimal immersions with conformality $\lambda_{p} / m$.

To complete this process we consider the chain of $S O(m+1)$-modules

$$
S^{2}\left(\mathcal{H}_{m}^{p}\right) \supset \mathcal{E}_{m}^{p} \supset \mathcal{F}_{m}^{p}=\mathcal{F}_{m}^{p ; 1} \supset \mathcal{F}_{m}^{p ; 2} \supset \ldots \supset \mathcal{F}_{m}^{p ;[|p / 2|]-1}
$$

where each term is obtained from the decomposition of $S^{2}\left(\mathcal{H}_{m}^{p}\right)$ in (1) by restriction to the corresponding triangle in the sequence

$$
\triangle_{0}^{p} \supset \triangle_{1}^{p} \supset \triangle_{2}^{p} \supset \triangle_{3}^{p} \supset \ldots \supset \triangle_{[|p / 2|]}^{p}
$$

and $\triangle_{k}^{p}, k=0, \ldots,[|p / 2|]$, is the closed convex triangle with vertices $(2 k, 2 k),(p, p)$ and $(2(p-k), 2 k)$. ([|.|] is the greatest integer function.) Intersecting each term in (7) with $\mathcal{K}_{m}^{p}$ we obtain the sequence

$$
\mathcal{K}_{m}^{p} \supset \mathcal{L}_{m}^{p} \supset \mathcal{M}_{m}^{p}=\mathcal{M}_{m}^{p ; 1} \supset \mathcal{M}_{m}^{p ; 2} \supset \ldots \supset \mathcal{M}_{m}^{p ;[|p / 2|]-1} .
$$

The objective of this paper is to give a geometric characterization of the minimal immersions that are parametrized by $\mathcal{M}_{m}^{p ; k}$, for each $k=1, \ldots,[|p / 2|]-1$.

Let $f: S_{\kappa_{p}}^{m} \rightarrow S_{V}$ be an isometric minimal immersion. We say that $f$ is isotropic of order $k, 2 \leq k \leq p$, if, for all $2 \leq l \leq k$, we have

$$
\begin{aligned}
& \left\langle\beta_{l}(f)\left(X_{1}, \ldots, X_{l}\right), \beta_{l}(f)\left(X_{l+1}, \ldots, X_{2 l}\right)\right\rangle \\
& \quad=\left\langle\beta_{l}\left(f_{m, p}\right)\left(X_{1}, \ldots, X_{l}\right), \beta_{l}\left(f_{m, p}\right)\left(X_{l+1}, \ldots, X_{2 l}\right)\right\rangle,
\end{aligned}
$$

where $X_{1}, \ldots, X_{2 l} \in T\left(S_{\kappa_{p}}^{m}\right)$ and $\beta_{l}(f)$ is the $l$-th fundamental form of $f$ [11]. Note that it is customary to define the first fundamental form of $f$ as the differential of $f$. For $l=1,(8)$, that is "isotropy of order 1 ", means that $f$ is an isometric minimal immersion.

Theorem 1. Let $f: S_{\kappa_{p}}^{m} \rightarrow S_{V}$ be an isometric minimal immersion and assume that $f$ is isotropic of order $k$. If $p \leq 2 k+1$, then $f$ is congruent to the standard minimal immersion $f_{m, p}$. For $m \geq 4$ and $p \geq 2(k+1)$, the set of congruence classes of full isometric minimal immersions $f: S_{\kappa_{p}}^{m} \rightarrow S_{V}$ that are isotropic of order $k$ can be parametrized by the compact convex body $\mathcal{M}_{m}^{p ; k}$ whose linear span is the $S O(m+1)$-module $\mathcal{F}_{m}^{p ; k}$ with complexification having irreducible decomposition as

$$
\mathcal{F}_{m}^{p ; k}=\sum_{(u, v) \in \triangle_{k+1}^{p} ; u, v \text { even }} V_{m+1}^{(u, v, 0, \ldots, 0)}
$$

where $\triangle_{k+1}^{p} \subset \mathbf{R}^{2}$ is the closed convex triangle with vertices $(2(k+1), 2(k+1))$, $(p, p)$ and $(2(p-k-1), 2(k+1))$. 
Remark. For $m=3$ and $p \geq 2(k+1)$, we can only assert that the set of congruence classes of full isometric minimal immersions $f: S_{\kappa_{p}}^{3} \rightarrow S_{V}$ that are isotropic of order $k$ can be parametrized by a compact convex body that is a slice of $\mathcal{M}_{m}^{p}$ by an $S O(4)$-submodule of $\mathcal{F}_{3}^{p}$ which contains $\mathcal{F}_{3}^{p ; k}$.

\section{Preliminaries}

We endow $\mathcal{H}_{m}^{p}$, the $S O(m+1)$-module of spherical harmonics of order $p$ on $S^{m}$, with the normalized $L^{2}$-scalar product

$$
\left\langle h_{1}, h_{2}\right\rangle=\frac{N(m, p)+1}{\operatorname{vol} S^{m}} \int_{S^{m}} h_{1} h_{2} v_{S^{m}}, \quad h_{1}, h_{2} \in \mathcal{H}_{m}^{p},
$$

where $v_{S^{m}}$ is the volume form on $S^{m}$, vol $S^{m}$ is the volume of $S^{m}$, and

$$
N(m, p)+1=\operatorname{dim} \mathcal{H}_{m}^{p}=(2 p+m-1) \frac{(p+m-2) !}{p !(m-1) !}
$$

The standard harmonic $p$-homogeneous polynomial map $f_{m, p}: \mathbf{R}^{m+1} \rightarrow \mathcal{H}_{m}^{p}$ is defined by

$$
f_{m, p}(x)=\sum_{j=0}^{N(m, p)} f_{m, p}^{j}(x) f_{m, p}^{j}, \quad x=\left(x_{0}, \ldots, x_{m}\right) \in \mathbf{R}^{m+1},
$$

where $\left\{f_{m, p}^{j}\right\}_{j=0}^{N(m, p)} \subset \mathcal{H}_{m}^{p}$ is an orthonormal basis. Now, given a full harmonic $p$-homogeneous polynomial map $f: \mathbf{R}^{m+1} \rightarrow V$, we have $f=A \circ f_{m, p}$, where $A: \mathcal{H}_{m}^{p} \rightarrow V$ is linear and surjective. We define

$$
\langle f\rangle=A^{\top} A-I \in S^{2}\left(\mathcal{H}_{m}^{p}\right) .
$$

$\langle f\rangle$ depends only on the congruence class of $f$. The map that associates to the congruence class of $f$ the symmetric linear endomorphism $\langle f\rangle$ gives a 1-1 correspondence between the set of congruence classes of full harmonic $p$-homogeneous polynomial maps $f: \mathbf{R}^{m+1} \rightarrow V$, for various $V$, and the convex body

$$
\mathcal{K}_{m}^{p}=\left\{C \in S^{2}\left(\mathcal{H}_{m}^{p}\right) \mid C+I \geq 0\right\}
$$

in $S^{2}\left(\mathcal{H}_{m}^{p}\right)$. (Here $\geq 0$ means positive semidefinite.) This is because $A^{\top} A \geq 0$ for all $A$ and, for $C=\langle f\rangle$, the unique square root of $C+I$ defines $A$.

The map $f_{m, p}: \mathbf{R}^{m+1} \rightarrow \mathcal{H}_{m}^{p}$ is equivariant with respect to the homomorphism $\rho_{m, p}: S O(m+1) \rightarrow S O\left(\mathcal{H}_{m}^{p}\right)$ that is just the usual $S O(m+1)$-module structure on $\mathcal{H}_{m}^{p}$ (given by precomposing spherical harmonics with the inverse of the isometries of $\left.S^{m}\right)$. The induced action of $g \in S O(m+1)$ on $\mathcal{K}_{m}^{p}$ is given by

$$
\langle f\rangle \mapsto g \cdot\langle f\rangle=\left\langle f \circ g^{-1}\right\rangle=\rho_{m, p}(g)\langle f\rangle \rho_{m, p}\left(g^{-1}\right)
$$

and thus it is the restriction of the $S O(m+1)$-module structure on $S^{2}\left(\mathcal{H}_{m}^{p}\right)$ extended from that of $\mathcal{H}_{m}^{p}$. By equivariance, $f_{m, p}$ maps the unit sphere $S^{m}$ into a sphere in $\mathcal{H}_{m}^{p}$ which, due to the normalizing in (10), is also unit. Restricting, we obtain the standard $p$-eigenmap $f_{m, p}: S^{m} \rightarrow S_{\mathcal{H}_{m}^{p}}$. As noted in the introduction, a harmonic $p$-homogeneous polynomial map $f: \mathbf{R}^{m+1} \rightarrow V$ is (or, more precisely, restricts to) a p-eigenmap $f: S^{m} \rightarrow S_{V}$ iff it is spherical that is

$$
|f(x)|^{2}=|x|^{2 p},|x|^{2}=x_{0}^{2}+\ldots+x_{m}^{2}, x=\left(x_{0}, \ldots, x_{m}\right) \in \mathbf{R}^{m+1} .
$$


Setting $f=A \circ f_{m, p}$ with $\langle f\rangle=A^{\top} A-I$ as above and noting that $f_{m, p}$ satisfies (12), we can rewrite this condition as

$$
|f(x)|^{2}-|x|^{2 p}=|f(x)|^{2}-\left|f_{m, p}(x)\right|^{2}=\left\langle\langle f\rangle f_{m, p}(x), f_{m, p}(x)\right\rangle=0, \quad x \in \mathbf{R}^{m+1} .
$$

Let $\mathcal{P}_{m+1}^{q}$ denote the $S O(m+1)$-module of $q$-homogeneous polynomials on $\mathbf{R}^{m+1}$ (with the same module structure as that of the spherical harmonics). Consider the $S O(m+1)$-module homomorphism

$$
\Psi_{m, p}^{0}: S^{2}\left(\mathcal{H}_{m}^{p}\right) \rightarrow \mathcal{P}_{m+1}^{2 p}
$$

defined by

$$
\Psi_{m, p}^{0}(C)=\left\langle C f_{m, p}, f_{m, p}\right\rangle, \quad C \in S^{2}\left(\mathcal{H}_{m}^{p}\right) .
$$

By (13), $\Psi_{m, p}^{0}(\langle f\rangle)=\left\langle\langle f\rangle f_{m, p}, f_{m, p}\right\rangle=0$ iff $f$ is spherical, that is $f: \mathbf{R}^{m+1} \rightarrow V$ restricts to a $p$-eigenmap $f: S^{m} \rightarrow S_{V}$. We obtain that setting

$$
\mathcal{E}_{m}^{p}=\operatorname{ker} \Psi_{m, p}^{0} \subset S^{2}\left(\mathcal{H}_{m}^{p}\right)
$$

and

$$
\mathcal{L}_{m}^{p}=\mathcal{K}_{m}^{p} \cap \mathcal{E}_{m}^{p}=\left\{C \in \mathcal{E}_{m}^{p} \mid C+I \geq 0\right\},
$$

the convex body $\mathcal{L}_{m}^{p}$ parametrizes the set of congruence classes of full $p$-eigenmaps $f: S^{m} \rightarrow S_{V}$, the parametrization being the same as for harmonic $p$-homogeneous polynomial maps above. Each endomorphism in $\mathcal{E}_{m}^{p}$ is traceless [5] so that, unlike $\mathcal{K}_{m}^{p}, \mathcal{L}_{m}^{p}$ is compact. It is easy to see that $\Psi^{0}$ maps onto $\mathcal{P}_{m+1}^{2 p}$ (by evaluating it on the harmonic $p$-homogeneous function $H\left(x_{m}^{p}\right): \mathbf{R}^{m+1} \rightarrow \mathbf{R}$ that represents the unique zonal in $\mathcal{H}_{m}^{p}$, where $H: \mathcal{P}_{m+1}^{q} \rightarrow \mathcal{H}_{m}^{q}$ is the harmonic projection operator [10]). Thus $\mathcal{E}_{m}^{p}=S^{2}\left(\mathcal{H}_{m}^{p}\right) / \mathcal{P}_{m+1}^{2 p}$. The canonical decomposition of polynomials into spherical harmonics [10] gives

$$
\mathcal{P}_{m+1}^{2 p}=\sum_{l=0}^{p} \mathcal{H}_{m}^{2 l} \cdot|x|^{2(p-l)}
$$

so that, as $S O(m+1)$-modules:

$$
\mathcal{P}_{m+1}^{2 p}=\sum_{l=0}^{p} \mathcal{H}_{m}^{2 l}=\sum_{l=0}^{p} V_{m+1}^{(2 l, 0, \ldots, 0)}
$$

where we follow the usual (mal)practice of denoting a representation and its complexification by the same symbol (for sums of absolutely irreducible representations). Going back to the decomposition of $S^{2}\left(\mathcal{H}_{m}^{p}\right)$ in (1), we see that (4) follows.

We now wish to use (an upgraded version of) this argument to obtain (3) for minimal immersions. In the following brief account we summarize some of the results in [9].

As noted in the introduction, a map $f: S_{\kappa_{p}}^{m} \rightarrow S_{V}$ is an isometric minimal immersion iff, scaling the metric on the source to curvature $1, f: S^{m} \rightarrow S_{V}$ is a $p$-eigenmap that is homothetic with homothety $\lambda_{p} / m$ :

$$
\left\langle f_{*}(X), f_{*}(Y)\right\rangle=\left(\lambda_{p} / m\right)\langle X, Y\rangle, \quad X, Y \in T\left(S^{m}\right) .
$$

Since $S^{m}$ has the property that, at each point of $S^{m}$, the isotropy subgroup of $S O(m+1)$ acts transitively on the unit sphere of the tangent space, by equivariance, $f_{m, p}: S^{m} \rightarrow S_{\mathcal{H}_{m}^{p}}$ satisfies (14) and thus it is minimal. $f_{m, p}: S^{m} \rightarrow S_{\mathcal{H}_{m}^{p}}$ is called 
the standard minimal immersion. The homothety condition (14) for $f$ can be rewritten as

$$
\left\langle f_{*}(X), f_{*}(Y)\right\rangle=\left\langle\left(f_{m, p}\right)_{*}(X),\left(f_{m, p}\right)_{*}(Y)\right\rangle, \quad X, Y \in T\left(S^{m}\right) .
$$

Given a $p$-eigenmap $f: S^{m} \rightarrow S_{V}$, we introduce the 2 -tensor $\Psi_{m, p}^{1}(f)$ on $S^{m}$ by

$$
\Psi_{m, p}^{1}(f)(X, Y)=\left\langle f_{*}(X), f_{*}(Y)\right\rangle-\left\langle\left(f_{m, p}\right)_{*}(X),\left(f_{m, p}\right)_{*}(Y)\right\rangle, \quad X, Y \in T\left(S^{m}\right) .
$$

By (15), $f$ is homothetic (and thereby minimal) iff $\Psi_{m, p}^{1}(f)=0$. The tensor $\Psi_{m, p}^{1}(f)$ depends only on the congruence class $\langle f\rangle$ of $f$. In fact, rewriting the homothety condition (15), we obtain

$$
\Psi_{p, m}^{1}(f)(X, Y)=\Psi_{m, p}^{1}(\langle f\rangle)(X, Y)=\left\langle X\langle f\rangle f_{p}, Y f_{p}\right\rangle,
$$

where $X, Y \in T\left(S^{m}\right)$ act on the vector valued functions as differentiations. The goal is to extend this to $\mathbf{R}^{m+1}$ and write it as the kernel of a homomorphism, defined on $\mathcal{E}_{m}^{p}$, but (15) gives infinitely many conditions because of the presence of the tangent vectors. We remedy this by restricting ourselves to conformal fields $X^{a}$, parametrized by a vector $a \in \mathbf{R}^{m+1}=\mathcal{H}_{m}^{1}$, defined by

$$
\left(X_{x}^{a}\right)^{\sim}=a-\frac{\langle a, x\rangle}{|x|^{2}} x, x \in \mathbf{R}^{m+1},
$$

where ${ }^{-}$denotes translation of vectors to the origin. Given a $p$-eigenmap $f: S^{m} \rightarrow$ $S_{V}$ thought to be a spherical $p$-homogeneous harmonic polynomial map $f: \mathbf{R}^{m+1} \rightarrow$ $V$, we now define

$$
\begin{aligned}
\Psi_{m, p}^{1}(f)\left(X^{a}, X^{b}\right) & =\left\langle f_{*}\left(X^{a}\right), f_{*}\left(X^{b}\right)\right\rangle-\left\langle\left(f_{m, p}\right)_{*}\left(X^{a}\right),\left(f_{m, p}\right)_{*}\left(X^{b}\right)\right\rangle \\
& =\left\langle f_{*}\left(X^{a}\right), f_{*}\left(X^{b}\right)\right\rangle-\left(\lambda_{p} / m\right)\left\langle X^{a}, X^{b}\right\rangle|x|^{2(p-1)} .
\end{aligned}
$$

The right hand side, as a function on $\mathbf{R}^{m+1}$, is actually a polynomial; an element of $\mathcal{P}_{m+1}^{2(p-1)}[9]$. More precisely, we have

$$
\begin{aligned}
\Psi_{m, p}^{1}(f)\left(X^{a}, X^{b}\right) & =\left\langle\partial_{a} f, \partial_{b} f\right\rangle-\left\langle\partial_{a} f_{m, p}, \partial_{b} f_{m, p}\right\rangle \\
& =\left\langle\partial_{a}\langle f\rangle f_{m, p}, \partial_{b} f_{m, p}\right\rangle,
\end{aligned}
$$

where the partial derivatives with vector parameters are directional derivatives in the corresponding direction. In fact, by (18), as differential operators,

$$
X_{x}^{a}=\partial_{a}-\frac{\langle a, x\rangle}{|x|^{2}} \partial_{x}
$$

so that the principal part of $X^{a}$ is $\partial_{a}$ since the radial derivative $\partial_{x}$ acts on homogeneous polynomials by a constant multiple of the identity. The lower order terms give no contribution in (20) since the corresponding scalar products are independent of $f$ and thereby cancel when taking the difference.

The obvious extension of (20) is to define, for $C \in \mathcal{E}_{m}^{p}, \Psi_{m, p}^{1}(C)$ as a map $\mathcal{H}_{m}^{1} \times \mathcal{H}_{m}^{1} \rightarrow \mathcal{P}_{m+1}^{2(p-1)}$ by

$$
\Psi_{m, p}^{1}(C)(a, b)=\left\langle\partial_{a} C f_{m, p}, \partial_{b} f_{m, p}\right\rangle .
$$

$\Psi_{m, p}^{1}$ is symmetric, that is actually defined on $S^{2}\left(\mathcal{H}_{m}^{1}\right)$ and traceless, that is zero on the trivial summand in $S^{2}\left(\mathcal{H}_{m}^{1}\right)=\mathcal{H}_{m}^{0} \oplus \mathcal{H}_{m}^{2}$. Restricting, it becomes a linear map 
$\Psi_{m, p}^{1}(C): \mathcal{H}_{m}^{2} \rightarrow \mathcal{P}_{m+1}^{2(p-1)}$, or equivalently, an element $\Psi_{m, p}^{1}(C) \in \mathcal{P}_{m+1}^{2(p-1)} \otimes \mathcal{H}_{m}^{2}$ Varying $C \in \mathcal{E}_{m}^{p}$, we obtain the linear map

$$
\Psi_{m, p}^{1}: \mathcal{E}_{m}^{p} \rightarrow \mathcal{P}_{m+1}^{2(p-1)} \otimes \mathcal{H}_{m}^{2}
$$

which is a homomorphism of $S O(m+1)$-modules (since the entire construction is equivariant under $S O(m+1)$ ). Since the conformal fields span (pointwise) each tangent space, we have

$$
\mathcal{F}_{m}^{p}=\operatorname{ker} \Psi_{m, p}^{1}
$$

As $S O(m+1)$-modules

$$
\mathcal{P}_{m+1}^{2(p-1)} \otimes \mathcal{H}_{m}^{2}=\sum_{l=0}^{p-1} \mathcal{H}_{m}^{2 l} \otimes \mathcal{H}_{m}^{2}
$$

so that the recurrence formula (2) immediately gives that the only common irreducible components in $\mathcal{E}_{m}^{p}$ and $\mathcal{P}^{2(p-1)} \otimes \mathcal{H}_{m}^{2}$ are

$$
V_{m+1}^{(2 l, 2,0, \ldots, 0)}, \quad l=1, \ldots, p-1 .
$$

In (4), these correspond to the base of $\triangle_{1}^{p}$. The DoCarmo-Wallach lower bound $[5,11]$ follows immediately. The proof of $(3)$ now amounts to show that $\Psi_{m, p}^{1}$ is nonzero (and hence isomorphic) on each component in (22). This (most technical part) is carried out by a (2-step) induction in the use of the "degree raising and lowering" operators that will also play a crucial role here. We give a brief account on these operators as follows. (For details and proofs, cf. [9].)

Let $H: \mathcal{P}_{m+1}^{q} \rightarrow \mathcal{H}_{m}^{q}$ denote the harmonic projection operator [10]. Given a harmonic $p$-homogeneous polynomial map $f: S^{m} \rightarrow S_{V}$, we define the harmonic $(p \pm 1)$-homogeneous polynomial maps

$$
f^{ \pm}: \mathbf{R}^{m+1} \rightarrow V \otimes \mathcal{H}_{m}^{1}
$$

by

$$
f^{+}=\sqrt{\lambda_{2 p} /\left(2 \lambda_{p}\right)} \sum_{i=0}^{m} H\left(x_{i} f\right) \otimes y_{i} \text { and } f^{-}=\sqrt{2 / \lambda_{2 p}} \sum_{i=0}^{m} \frac{\partial f}{\partial x_{i}} \otimes y_{i} .
$$

Taking congruence classes, the correspondences $f \mapsto f^{ \pm}$are the restrictions of $S O(m+1)$-module homomorphisms

$$
\Phi_{m, p}^{ \pm}: S^{2}\left(\mathcal{H}_{m}^{p}\right) \rightarrow S^{2}\left(\mathcal{H}_{m}^{p \pm 1}\right)
$$

that can be described explicitly as follows. By the recurrence formula $(2), \mathcal{H}_{m}^{p \pm 1}$ are both $S O(m+1)$-submodules of $\mathcal{H}_{m}^{p} \otimes \mathcal{H}_{m}^{1}$. More explicitly, the corresponding $S O(m+1)$-module monomorphisms

$$
\iota_{ \pm}: \mathcal{H}_{m}^{p \pm 1} \rightarrow \mathcal{H}_{m}^{p} \otimes \mathcal{H}_{m}^{1}
$$

are given by

$$
\iota_{-}\left(h^{\prime}\right)=c^{-} \sum_{i=0}^{m} H\left(x_{i} h^{\prime}\right) \otimes y_{i}, \quad h^{\prime} \in \mathcal{H}_{m}^{p-1}
$$

and

$$
\iota_{+}\left(h^{\prime \prime}\right)=c^{+} \sum_{i=0}^{m} \frac{\partial h^{\prime \prime}}{\partial x_{i}} \otimes y_{i}, \quad h^{\prime \prime} \in \mathcal{H}_{m}^{p+1}
$$


The value of the normalizing constants $c^{ \pm}$is given by the requirement that

$$
\left(\iota^{ \pm}\right)^{\top} \circ \iota^{ \pm}=I \text {. }
$$

Now

$$
\Phi_{m, p}^{ \pm}(C)=\iota_{ \pm}^{\top} \circ(C \otimes I) \circ \iota_{ \pm}, \quad C \in S^{2}\left(\mathcal{H}_{m}^{p}\right)
$$

and, as noted above

$$
\Phi_{m, p}^{ \pm}(\langle f\rangle)=\left\langle f^{ \pm}\right\rangle
$$

for any harmonic $p$-homogeneous polynomial map $f: \mathbf{R}^{m+1} \rightarrow V$. Note finally [9] that

$$
\iota_{ \pm}\left(f_{m, p \pm 1}(x)\right)=f_{m, p}^{ \pm}(x), x \in S^{m} .
$$

$\Phi_{m, p}^{+}$is injective and $\Phi_{m, p}^{-}$, being, up to a constant multiple, the transpose of $\Phi_{m, p}^{+}$, is surjective. In terms of the decomposition of $S^{2}\left(\mathcal{H}_{m}^{p}\right)$ in $(1), \Phi_{m, p}^{+}$corresponds to the inclusion of the triangles $\triangle_{0}^{p} \subset \triangle_{0}^{p+1}$, and $\Phi_{m, p}^{-}$to "slicing off" the northeast side of $\triangle_{0}^{p}$ to get $\triangle_{0}^{p-1}$. Equivalently

$$
\operatorname{coker} \Phi_{m, p}^{+}=\operatorname{ker} \Phi_{m, p+1}^{-}=\sum_{i=0}^{[|(p+1) / 2|]} V_{m+1}^{(2(p+1-i), 2 i, 0, \ldots, 0)} .
$$

The degree raising and lowering operators $f \mapsto f^{ \pm}$preserve sphericality, conformality [9] and, as we will see later, isotropy for all order. In particular, degree raising defines equivariant imbeddings $\mathcal{K}_{m}^{p} \rightarrow \mathcal{K}_{m}^{p+1}, \mathcal{L}_{m}^{p} \rightarrow \mathcal{L}_{m}^{p+1}$ and $\mathcal{M}_{m}^{p} \rightarrow \mathcal{M}_{m}^{p+1}$ (and similarly for isotropy of all order).

Remark. Degree lowering appears implicitly in the work of DoCarmo-Wallach $[5,11]$ in the form of the differential operator

$$
\sum_{i=0}^{m} \partial^{2} / \partial x_{i} \partial y_{i}: \mathcal{H}_{m}^{p} \otimes \mathcal{H}_{m}^{q} \rightarrow \mathcal{H}_{m}^{p-1} \otimes \mathcal{H}_{m}^{q-1}
$$

as a technical tool to derive the recurrence formula in (2).

\section{ISOTROPY}

Let $f: S^{m} \rightarrow S_{V}$ be a minimal immersion with homothety $\lambda_{p} / m$. (From now on we scale the metric on the source to curvature 1.) Let $\beta_{k}(f), k=1, \ldots, p$, denote the $k$-th fundamental form of $f$. Recall [11] that $\beta_{k}(f)$ and the $k$-th osculating bundle $\mathcal{O}_{f}^{k}$ are defined inductively on a (maximal) open and dense set $D_{f} \subset S^{m}$ as follows. For $x \in D_{f}, \beta_{k}(f): S^{k}\left(T_{x}\left(S^{m}\right)\right) \rightarrow \mathcal{O}_{f ; x}^{k}$ is a linear map of the $k$-th symmetric power of the tangent space $T_{x}\left(S^{m}\right)$ onto the fibre $\mathcal{O}_{f ; x}^{k}$ of $\mathcal{O}_{f}^{k}$ at $x$. The latter is called the $k$-th osculating space of $f$ at $x$. For $k=1, \beta_{1}(f)=f_{*}$ is defined on $D_{f}^{1}=S^{m}$ and, for $x \in D_{f}^{1}$, the first osculating space $\mathcal{O}_{f ; x}^{1}$ at $x$ is the image of $\beta_{1}(f)_{x}$. The general induction is given by

$$
\begin{aligned}
\beta_{k}(f)\left(X_{1}, \ldots, X_{k}\right)= & \left(\nabla_{X_{k}} \beta_{k-1}(f)\right)\left(X_{1}, \ldots, X_{k-1}\right)^{\perp_{k-1}}, \\
& X_{1}, \ldots, X_{k} \in T_{x}\left(S^{m}\right), x \in D_{f}^{k-1},
\end{aligned}
$$

where $\perp_{k-1}$ is the orthogonal projection with kernel $\mathcal{O}_{f ; x}^{0} \oplus \mathcal{O}_{f ; x}^{1} \oplus \ldots \oplus \mathcal{O}_{f ; x}^{k-1}$, $\mathcal{O}_{f ; x}^{0}=\mathbf{R} \cdot f(x)$, and $D_{f}^{k}$ is the set of points $x \in D_{f}^{k-1}$ at which the image $\mathcal{O}_{f ; x}^{k}$ of $\beta_{k}(f)_{x}$ has maximal dimension. We define $D_{f}=\bigcap_{k=0}^{p} D_{f}^{k}$. The largest $k$ for which 
$\beta_{k}(f)$ is nonvanishing is called the geometric degree $d_{f}$ of $f$. We have $d_{f} \leq d_{f_{p}}=p$ and, by equivariance, $D_{f_{p}}=S^{m}$.

For $k=1$, the first fundamental form $\beta_{1}(f)$ makes sense for a $p$-eigenmap $f$ : $S^{m} \rightarrow S_{V}$ and will be interpreted as the differential of $f$. Finally, for a harmonic $p$-homogeneous polynomial map $f: \mathbf{R}^{m+1} \rightarrow V$, the 0 -th fundamental form $\beta_{0}(f)$ (with no argument) will be interpreted as $f$ itself.

For $0 \leq k \leq p$, we introduce the $(2 k)$-tensor $\Psi_{m, p}^{k}(f)$ on $D_{f} \subset S^{m}$ by

$$
\begin{aligned}
\Psi_{m, p}^{k}(f)\left(X_{1}, \ldots, X_{2 k}\right)= & \left\langle\beta_{k}(f)\left(X_{1}, \ldots, X_{k}\right), \beta_{k}(f)\left(X_{k+1}, \ldots, X_{2 k}\right)\right\rangle \\
& -\left\langle\beta_{k}\left(f_{m, p}\right)\left(X_{1}, \ldots, X_{k}\right), \beta_{k}\left(f_{m, p}\right)\left(X_{k+1}, \ldots, X_{2 k}\right)\right\rangle
\end{aligned}
$$

where $X_{1}, \ldots, X_{2 k} \in T\left(S^{m}\right)$. Clearly, for $k=1$ this definition agrees with (16) for $p$-eigenmaps $f: S^{m} \rightarrow S_{V}$. Moreover, for a harmonic $p$-homogeneous polynomial map $f: \mathbf{R}^{m+1} \rightarrow V$, we have $\beta_{0}(f)=f$, so that this definition, for $k=0$, agrees with the one introduced in Section 2.

A minimal immersion $f: S^{m} \rightarrow S_{V}$ with homothety $\lambda_{p} / m$ is said to be isotropic of order $k, 2 \leq k \leq p$ if

$$
\Psi_{m, p}^{2}(f)=\ldots=\Psi_{m, p}^{k}(f)=0
$$

Using the extended meaning of the fundamental forms for $k=0,1$, we see that a p-eigenmap $f: S^{m} \rightarrow S_{V}$ is isotropic of order 1 iff it is a minimal immersion with homothety $\lambda_{p} / m$ and a harmonic $p$-homogeneous polynomial map $f: \mathbf{R}^{m+1} \rightarrow V$ is isotropic of order $0 \mathrm{iff}$ it is spherical. Thus, the order of isotropy (in the appropriate category) is defined for all $0 \leq k \leq p$.

As before, we now restrict ourselves to conformal vector fields $X^{a}$ parametrized by $a \in \mathcal{H}_{m}^{1}$. Let $f: S^{m} \rightarrow S_{V}$ any minimal immersion with homothety $\lambda_{p} / m$. We claim that, on $D_{f}$, we have

$$
\begin{aligned}
\beta_{k}(f)\left(X^{a_{1}}, \ldots, X^{a_{k}}\right)^{\llcorner}= & \partial_{a_{1}} \ldots \partial_{a_{k}} f+\text { lower order terms, } \\
& a_{1}, \ldots, a_{k} \in \mathcal{H}_{m}^{1}, k \geq 1,
\end{aligned}
$$

where means translating vectors to the origin. We prove this by induction with respect to $k$. The basic step, $k=1$, is clear:

$$
\beta_{1}(f)\left(X^{a}\right)^{\llcorner}=f_{*}\left(X^{a}\right)^{\llcorner}=X^{a}(f)=\partial_{a} f+p \frac{\langle a, x\rangle}{|x|^{2}} f,
$$

where we used (21) and that the radial derivative $\partial_{x}$ acts on the $p$-homogeneous polynomial map $f$ as multiplication by $p$. For the general induction step $k-1 \Rightarrow k$, we recall from the inductive definition of higher fundamental forms that, apart from the orthogonal projection to the previous osculating subbundle that is obtained (by the induction hypothesis) by subtracting an appropriate multiple of lower order terms, $\beta_{k}(f)\left(X^{a_{1}}, \ldots, X^{a_{k}}\right)^{-}$is the covariant derivative

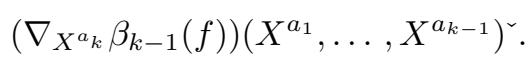

The principal term of this is the same as that of $X^{a_{k}}\left(\beta_{k-1}\left(X^{a_{1}}, \ldots, X^{a_{k-1}}\right)^{\smile}\right)$. The principal term in $X^{a_{k}}$ is $\partial_{a_{k}}$ and, by the induction hypothesis, the principal term in $\beta_{k-1}(f)\left(X^{a_{1}}, \ldots, X^{a_{k-1}}\right)^{-}$is $\partial_{a_{1}} \ldots \partial_{a_{k-1}} f$. (26) follows.

Remark. Let $f: S^{m} \rightarrow S_{V}$ be a minimal immersion with homothety $\lambda_{p} / m$ and set $f=A \circ f_{m, p}$. Then we have

$$
A \beta_{l}\left(f_{m, p}\right)\left(X^{a_{1}}, \ldots, X^{a_{2 l}}\right)^{\llcorner}=\beta_{l}(f)\left(X^{a_{1}}, \ldots, X^{a_{2 l}}\right)^{\llcorner}, \quad 1 \leq l \leq p .
$$


Thus, $A$ maps $\check{\mathcal{O}}_{f_{m, p} ; x}^{l}$ onto $\check{\mathcal{O}}_{f ; x}^{l}$. Now isotropy of order $k$ just says that $A$ defines a fibrewise isometry between the $l$-th osculating spaces, $1 \leq l \leq k$. We obtain that order $k$ isotropy implies that the osculating bundles $\mathcal{O}_{f}^{l}$ and $\overline{\mathcal{O}}_{f_{m, p}}^{l}, 1 \leq l \leq k$, are isomorphic with a fibrewise isometry. As $S O(m)$-modules [5, 11], $\mathcal{O}_{f_{m, p} ; o}^{l}=\mathcal{H}_{m-1}^{l}$, $0 \leq l \leq p$, so that order $k$ isotropy gives a lower bound on the codimension of the minimal immersion.

We now evaluate $\Psi_{m, p}^{k}(f)$ in (24) on conformal fields $X^{a_{1}}, \ldots, X^{a_{2 k}}, a_{1}, \ldots, a_{2 k}$ $\in \mathcal{H}_{m}^{1}$. Retaining only the top term in (26) for the $k$-th fundamental forms of $f$ and $f_{m, p}$, the difference of the scalar products in (24) gives the term

$$
\begin{aligned}
\left\langle\partial_{a_{1}} \ldots \partial_{a_{k}} f, \partial_{a_{k+1}} \ldots \partial_{a_{2 k}} f\right\rangle & -\left\langle\partial_{a_{1}} \ldots \partial_{a_{k}} f_{m, p}, \partial_{a_{k+1}} \ldots \partial_{a_{2 k}} f_{m, p}\right\rangle \\
& =\left\langle\partial_{a_{1}} \ldots \partial_{a_{k}}\langle f\rangle f_{m, p}, \partial_{a_{k+1}} \ldots \partial_{a_{2 k}} f_{m, p}\right\rangle .
\end{aligned}
$$

In view of this, for $C \in S^{2}\left(\mathcal{H}_{m}^{p}\right)$ and $0 \leq k \leq p$, we define

$$
\Psi_{m, p}^{k}(C)\left(a_{1}, \ldots, a_{2 k}\right)=\left\langle\partial_{a_{1}} \ldots \partial_{a_{k}} C f_{m, p}, \partial_{a_{k+1}} \ldots \partial_{a_{2 k}} f_{m, p}\right\rangle,
$$

for $a_{1}, \ldots, a_{2 k} \in \mathcal{H}_{m}^{1}$. Note that $(28)$ is a $2(p-k)$-homogeneous polynomial on $\mathbf{R}^{m+1}$, that is an element of $\mathcal{P}_{m+1}^{2(p-k)}$. In the following crucial lemma we use standard multi-index notation; that is, for $I=\left\{i_{1}, \ldots, i_{l}\right\}$, we set $|I|=l$ and $\partial_{a_{I}}=\partial_{a_{1}} \ldots \partial_{a_{l}}$.

Inductive Lemma. Given $C \in S^{2}\left(\mathcal{H}_{m}^{p}\right)$ and $1 \leq k \leq p$, assume that

$$
\Psi_{p, m}^{0}(C)=\Psi_{p, m}^{1}(C)=\ldots=\Psi_{p, m}^{k-1}(C)=0 .
$$

Then we have

$$
\left\langle\partial_{a_{I}} C f_{m, p}, \partial_{a_{J}} f_{m, p}\right\rangle=0
$$

for all $I$ and $J$ with $0 \leq|I|+|J| \leq 2 k-1, I, J \subset\{1, \ldots, 2 k\}$; and

$$
\Psi_{m, p}^{k}(C)\left(a_{1}, \ldots, a_{2 k}\right)=(-1)^{(|I|-|J|) / 2}\left\langle\partial_{a_{I}} C f_{m, p}, \partial_{a_{J}} f_{m, p}\right\rangle
$$

for all $I$ and $J$ disjoint with $I \cup J=\{1, \ldots, 2 k\}$.

Proof. We use induction with respect to $k$. For $k=1$, the assumption says that

$$
\Psi_{m, p}^{0}(C)=\left\langle C f_{m, p}, f_{m, p}\right\rangle=0
$$

Differentiating and using symmetry of $C$, this implies that

$$
\left\langle\partial_{a} C f_{m, p}, f_{m, p}\right\rangle=\left\langle C f_{m, p}, \partial_{a} f_{m, p}\right\rangle=0
$$

and (29) follows. Finally, differentiating this again, we obtain

$$
\Psi_{m, p}^{1}(C)(a, b)=\left\langle\partial_{a} C f_{m, p}, \partial_{b} f_{m, p}\right\rangle=-\left\langle\partial_{a} \partial_{b} C f_{m, p}, f_{m, p}\right\rangle
$$

and (30) follows. For the general induction step $k-1 \Rightarrow k$, we introduce the notation

$$
F(I ; J)=F\left(i_{1}, \ldots, i_{r} ; i_{r+1}, \ldots, i_{r+s}\right)=\left\langle\partial_{a_{I}} C f_{m, p}, \partial_{a_{J}} f_{m, p}\right\rangle,
$$

where $I=\left\{i_{1}, \ldots, i_{r}\right\}$ and $J=\left\{i_{r+1}, \ldots, i_{r+s}\right\}$. By symmetry of $C$, we have $F(I, J)=F(J, I)$ so that we can write $F(I ; \emptyset)=F(I)$ and $F(\emptyset ; J)=F(J)$. By the induction hypothesis,

$$
F\left(i_{1}, \ldots, i_{r} ; i_{r+1}, \ldots, i_{r+s}\right)=0, \quad 0 \leq r+s \leq 2 k-2,
$$

in particular,

$$
F\left(i_{1}, \ldots, i_{r} ; i_{r+1}, \ldots, i_{2 k-2}\right)=0, \quad 0 \leq r \leq 2 k-2 .
$$


Differentiating this at the direction $a_{2 k-1}$, we obtain

$$
\begin{gathered}
F\left(i_{1}, \ldots, i_{r} ; i_{r+1}, \ldots, i_{2 k-2}, i_{2 k-1}\right)=-F\left(i_{1}, \ldots, i_{r}, i_{2 k-1} ; i_{r+1}, \ldots, i_{2 k-2}\right), \\
0 \leq r \leq 2 k-2 .
\end{gathered}
$$

Using this repeatedly, we arrive at

$$
\begin{aligned}
& F\left(i_{1}, \ldots, i_{k-1} ; i_{k}, \ldots, i_{2 k-1}\right)=(-1)^{k} F\left(i_{1}, \ldots, i_{2 k-1}\right), \\
& F\left(i_{k}, \ldots, i_{2 k-1} ; i_{1}, \ldots, i_{k-1}\right)=(-1)^{k-1} F\left(i_{1}, \ldots, i_{2 k-1}\right) .
\end{aligned}
$$

These, however, are equal by symmetry, so that $F\left(i_{1}, \ldots, i_{2 k-1}\right)=0$. Using (31) repeatedly again, we obtain

$$
F\left(i_{1}, \ldots, i_{r} ; i_{r+1}, \ldots, i_{2 k-1}\right)=0, \quad r=0, \ldots, 2 k-1 .
$$

This is (29). Now (30) is obtained by differentiating (29).

Theorem 2. Let $f: S^{m} \rightarrow S_{V}$ be a p-eigenmap and assume that $f$ is isotropic of order $k-1, k \geq 1$. Then, we have

$$
\Psi_{m, p}^{k}(f)\left(X^{a_{1}}, \ldots, X^{a_{2 k}}\right)=\Psi_{m, p}^{k}(\langle f\rangle)\left(a_{1}, \ldots, a_{2 k}\right), \quad a_{1}, \ldots, a_{2 k} \in \mathcal{H}_{m}^{1}
$$

We prove Theorem 2 by induction with respect to $k$. The basic step $k=1$ was done in Section 2, cf. (20). Now let $k \geq 2$ and perform the general induction step $0, \ldots, k-1 \Rightarrow k$. Assume that $f$ is isotropic of order $k-1$ that is

$$
\Psi_{m, p}^{0}(f)=\ldots=\Psi_{m, p}^{k-1}(f)=0 .
$$

Since $f$ is also isotropic of order $\leq k-2$ the induction hypothesis gives

$$
\Psi_{m, p}^{l}(f)\left(X^{a_{1}}, \ldots, X^{a_{2 l}}\right)=\Psi_{m, p}^{l}(\langle f\rangle)\left(a_{1}, \ldots, a_{2 l}\right)=0, \quad 0 \leq l \leq k-1 .
$$

Combining (34) and (35), we obtain

$$
\Psi_{m, p}^{l}(\langle f\rangle)\left(a_{1}, \ldots, a_{2 l}\right)=0, \quad 0 \leq l \leq k-1 .
$$

These are precisely the assumptions of the Inductive Lemma. Consider now

$$
\Psi_{m, p}^{k}(f)\left(X^{a_{1}}, \ldots, X^{a_{2 k}}\right) .
$$

As noted above, using the expansion in (26) for $f$ and $f_{m, p}$ in (24), this can be written as

$$
\Psi_{m, p}^{k}(\langle f\rangle)\left(a_{1}, \ldots, a_{2 k}\right)
$$

plus a sum of terms (with functional entries) of type

$$
\left\langle\partial_{a_{I}} f, \partial_{a_{J}} f\right\rangle-\left\langle\partial_{a_{I}} f_{m, p}, \partial_{a_{J}} f_{m, p}\right\rangle=\left\langle\partial_{a_{I}} C f_{m, p}, \partial_{a_{J}} f_{m, p}\right\rangle,
$$

where $I$ and $J$ satisfy $|I|+|J| \leq 2 k-1$. By the Inductive Lemma, these are all zero. Theorem 2 follows.

Corollary 1. Let $f: S^{m} \rightarrow S_{V}$ be a p-eigenmap. Then $f$ is isotropic of order $k$, iff

$$
\Psi_{m, p}^{0}(\langle f\rangle)=\ldots=\Psi_{m, p}^{k}(\langle f\rangle)=0 .
$$

Proof. By Theorem 2, we need only to prove that (37) implies isotropy of order $k$. Since the conformal fields span each tangent space, it is enough to show that

$$
\Psi_{m, p}^{l}(f)\left(X^{a_{1}}, \ldots, X^{a_{2 l}}\right)=0, a_{1}, \ldots, a_{2 l} \in \mathcal{H}_{m}^{1}, 0 \leq l \leq k .
$$

We now observe that, in the proof of Theorem 2, in deriving (33) we used only (36) that is precisely (37) (with $k$ replaced by $k+1$ ).

We are now able to prove rigidity; that is the first statement of Theorem 1. 
Corollary 2. Let $f: S^{m} \rightarrow S_{V}$ be a minimal immersion with homothety $\lambda_{p} / m$ and assume that $f$ is isotropic of order $k$. If $p \leq 2 k+1$ then $f$ is congruent to $f_{m, p}$.

Proof. Let $C=\langle f\rangle$. By Corollary 1

$$
\Psi_{m, p}^{0}(C)=\ldots=\Psi_{m, p}^{k}(C)=0 .
$$

Let $p \leq 2 k+1$. By (29) of the Inductive Lemma (with $k$ replaced by $k+1$ ), we have

$$
\left\langle\partial_{a_{1}} \ldots \partial_{a_{p}} C f_{m, p}, f_{m, p}\right\rangle=0, \quad a_{1}, \ldots, a_{p} \in \mathcal{H}_{m}^{1} .
$$

The first vector in the scalar product is constant since the degree of $f_{p}$ is $p$. On the other hand, $f_{m, p}$ is full so that

$$
\partial_{a_{1}} \ldots \partial_{a_{p}} C f_{m, p}=0 .
$$

Since this is valid for all $a_{1}, \ldots, a_{p}$, we can remove them and obtain

$$
C f_{m, p}=0 \text {. }
$$

Again by fullness, this means that $C=0$ so that $f$ is standard.

\section{The Main Induction SteP}

We now return to the main line and, proceeding inductively with respect to the order of isotropy, we let $p \geq 2(k+1)$ and assume that the set of congruence classes of full minimal immersions $f: S^{m} \rightarrow S_{V}$ that are isotropic of order $k-1$ are parametrized by $\mathcal{M}_{m}^{p ; k-1}$ with linear span $\mathcal{F}_{m}^{p ; k-1}$. We refer to this as the main induction hypothesis. It amounts geometrically to

$$
\mathcal{F}_{m}^{p ; k-1}=\left\{C \in S^{2}\left(\mathcal{H}_{m}^{p}\right) \mid \Psi_{m, p}^{0}(C)=\ldots=\Psi_{m, p}^{k-1}(C)=0\right\}
$$

and algebraically to

$$
\mathcal{F}_{m}^{p ; k-1}=\sum_{(u, v) \in \triangle_{k}^{p} ; u, v \text { even }} V_{m+1}^{(u, v, 0, \ldots, 0)} .
$$

Given $C \in \mathcal{F}_{m}^{p, k-1},(30)$ of the Inductive Lemma applies (with $J=\emptyset$ ) yielding that

$$
\Psi_{m, p}^{k}(C)\left(a_{1}, \ldots, a_{2 k}\right)
$$

is symmetric in all vector variables and can therefore be considered as a linear map

$$
\Psi_{m, p}^{k}(C): \mathcal{P}_{m+1}^{2 k} \rightarrow \mathcal{P}_{m+1}^{2(p-k)} .
$$

By harmonicity of $f_{m, p}$, contraction of $\Psi_{m, p}^{k}(C)\left(a_{1}, \ldots, a_{2 k}\right)$ with respect to any two vector variables is zero. This means that $\Psi_{m, p}^{k}(C)$, considered on $\mathcal{P}_{m+1}^{2 k}$, is zero on multiples of $|x|^{2}$ so that no information is lost by restricting it to $\mathcal{H}_{m}^{2 k}$; the orthogonal complement of $\mathcal{P}_{m+1}^{2(k-1)} \cdot|x|^{2}$ in $\mathcal{P}_{m+1}^{2 k}$ :

$$
\Psi_{m, p}^{k}(C): \mathcal{H}_{m}^{2 k} \rightarrow \mathcal{P}_{m+1}^{2(p-k)} .
$$

Equivalently, $\Psi_{m, p}^{k}(C)$ can be thought of as an element of $\mathcal{P}_{m+1}^{2(p-k)} \otimes \mathcal{H}_{m}^{2 k}$. Varying $C$ within $\mathcal{F}_{m}^{p ; k-1}$ we obtain the linear map

$$
\Psi_{m, p}^{k}: \mathcal{F}_{m}^{p ; k-1} \rightarrow \mathcal{P}_{m+1}^{2(p-k)} \otimes \mathcal{H}_{m}^{2 k}
$$

This is a homomorphism of $S O(m+1)$-modules. In fact, given $g \in S O(m+1)$, we have

$$
\Psi_{m, p}^{k}(g \cdot C)\left(g a_{1}, \ldots, g a_{2 k}\right)=\Psi_{m, p}^{k}(C)\left(a_{1}, \ldots, a_{2 k}\right) \circ g^{-1}, \quad a_{1}, \ldots, a_{2 k} \in \mathcal{H}_{m}^{1},
$$


where we use the transformation rule $g \cdot C=\rho_{m, p}(g) C \rho_{m, p}\left(g^{-1}\right)$ and equivariance of $f_{m, p}$.

Let $f: S^{m} \rightarrow S_{V}$ be a minimal immersion with homothety $\lambda_{p} / m$ and assume that $f$ is isotropic of order $k-1$. Then $\langle f\rangle \in \mathcal{M}_{m}^{p ; k-1}$ (main induction hypothesis) and Corollary 1 to Theorem 2 asserts that

$$
\Psi_{m, p}^{k}(\langle f\rangle)\left(a_{1}, \ldots, a_{2 k}\right), \quad a_{1}, \ldots, a_{2 k} \in \mathcal{H}_{m}^{1} .
$$

is zero iff $f$ is isotropic of order $k$. Thus, we obtain that the linear slice

$$
\overline{\mathcal{M}}_{m}^{p ; k-1}=\mathcal{M}_{m}^{p ; k-1} \cap \operatorname{ker} \Psi_{m, p}^{k}
$$

parametrizes the set of congruence classes of full minimal immersions $f: S^{m} \rightarrow S_{V}$ with homothety $\lambda_{p} / m$ that are isotropic of order $k$. We denote the linear span by

$$
\overline{\mathcal{F}}_{m}^{p ; k}=\operatorname{ker} \Psi_{m, p}^{k}
$$

The general induction step and thereby the proof of Theorem 1 will be accomplished if we show that $\overline{\mathcal{F}}_{m}^{p ; k}=\mathcal{F}_{m}^{p ; k}$ with $\mathcal{F}_{m}^{p ; k}$ defined in (9).

We now return to (40) and consider the canonical decomposition

$$
\mathcal{P}_{m+1}^{2(p-k)}=\sum_{l=0}^{p-k} \mathcal{H}_{m}^{2 l} \cdot|x|^{2(p-k-l)} .
$$

As $S O(m+1)$-modules, we have

$$
\mathcal{P}_{m+1}^{2(p-k)} \otimes \mathcal{H}_{m}^{2 k}=\sum_{l=0}^{p-k} \mathcal{H}_{m}^{2 l} \otimes \mathcal{H}_{m}^{2 k} .
$$

Decomposing the tensor products of spherical harmonics by the recurrence formula (2) we see that the only irreducible $S O(m+1)$-modules that are contained in both $\mathcal{F}_{m}^{p ; k-1}$ and $\mathcal{P}_{m+1}^{2(p-k)} \otimes \mathcal{H}_{m}^{2 k}$ are of the form

$$
V_{m+1}^{(2 l, 2 k, 0, \ldots, 0)}, \quad l=k, \ldots, p-k .
$$

These are precisely the ones corresponding to the base of the triangle $\triangle_{k}^{p}$. In particular, since they are disjoint from $\mathcal{F}_{m}^{p ; k}$, we immediately obtain

$$
\mathcal{F}_{m}^{p ; k} \subset \overline{\mathcal{F}}_{m}^{p ; k} \text {. }
$$

Moreover, equality holds iff

$$
V_{m+1}^{(2 l, 2 k, 0, \ldots, 0)} \not \subset \mathcal{F}_{m}^{p ; k}, \quad l=k, \ldots, p-k,
$$

and, by (41), this is certainly guaranteed by

$$
\Psi_{m, p}^{k} \mid V_{m+1}^{(2 l, 2 k, 0, \ldots, 0)} \neq 0, \quad l=k, \ldots, p-k .
$$

\section{Isotropy AND Degree RAising AND LOWERING}

The purpose of this section is to reduce (44) to the statement

$$
\Psi_{m, p}^{k} \mid V_{m+1}^{(2(p-k), 2 k, 0, \ldots, 0)} \neq 0, \quad p \geq 2(k+1),
$$

that is nonvanishing at the southeast vertex $(2(p-k), 2 k)$ of $\triangle_{k}^{p}$. The key is to show that isotropy is preserved under degree raising and lowering. This is an immediate consequence of the following: 
Theorem 3. Let $C \in S^{2}\left(\mathcal{H}_{m}^{p}\right)$ and assume that

$$
\Psi_{m, p}^{0}(C)=\ldots=\Psi_{m, p}^{k-1}(C)=0 .
$$

Then, for $a_{1}, \ldots, a_{2 k} \in \mathcal{H}_{m}^{1}$, we have

$$
\begin{aligned}
\Psi_{m, p+1}^{k}\left(\Phi_{m, p}^{+}(C)\right)\left(a_{1}, \ldots, a_{2 k}\right)= & \left(\lambda_{4 k} /(4 k)\right)\left(p / \lambda_{p}\right) \Psi_{m, p}^{k}(C)\left(a_{1}, \ldots, a_{2 k}\right)|x|^{2} \\
& +\left(p^{2} /\left(\lambda_{p} \lambda_{2 p}\right)\right) \triangle\left(\Psi_{m, p}^{k}(C)\left(a_{1}, \ldots, a_{2 k}\right)\right)|x|^{4}
\end{aligned}
$$

and

$$
\triangle\left(\Psi_{m, p}^{k}(C)\left(a_{1}, \ldots, a_{2 k}\right)\right)=\lambda_{2 p} \Psi_{m, p-1}^{k}\left(\Phi_{m, p}^{-}(C)\left(a_{1}, \ldots, a_{2 k}\right)\right) .
$$

Proof. Using the properties of degree raising, we compute

$$
\begin{aligned}
\Psi_{m, p+1}^{k} & \left(\Phi_{m, p}^{+}(C)\right)\left(a_{1}, \ldots, a_{2 k}\right)=\Psi_{m, p+1}^{k}\left(\iota_{+}^{\top}(C \otimes I) \iota_{+}\right)\left(a_{1}, \ldots, a_{2 k}\right) \\
& =\left\langle\partial_{a_{1}} \ldots \partial_{a_{k}}\left(\iota_{+}^{\top}(C \otimes I) \iota_{+}\right) f_{m, p+1}, \partial_{a_{1}} \ldots \partial_{a_{2 k}} f_{m, p+1}\right\rangle \\
& =\left\langle\partial_{a_{1}} \ldots \partial_{a_{k}}(C \otimes I) f_{m, p}^{+}, \partial_{a_{k+1}} \ldots \partial_{a_{2 k}} f_{m, p}^{+}\right\rangle \\
& =\left(\lambda_{2 p} /\left(2 \lambda_{p}\right)\right) \sum_{i=0}^{m}\left\langle\partial_{a_{1}} \ldots \partial_{a_{k}} H\left(x_{i} C f_{m, p}\right), \partial_{a_{k+1}} \ldots \partial_{a_{2 k}} H\left(x_{i} f_{m, p}\right)\right\rangle .
\end{aligned}
$$

Due to the harmonicity of $f_{m, p}$, the harmonic projection formula [10] specializes to

$$
H\left(x_{i} f_{m, p}\right)=x_{i} f_{m, p}-\frac{2 p}{\lambda_{2 p}} \frac{\partial f_{m, p}}{\partial x_{i}}|x|^{2} .
$$

We need to take the directional derivative of both sides of this $k$ times. For this purpose we use the product rule

$$
\partial_{a_{1}} \ldots \partial_{a_{k}}(u \cdot v)=\sum_{I, J} \partial_{a_{I}} u \cdot \partial_{a_{J}} v,
$$

where the summation is taken with respect to $I$ and $J$ such that $I \cap J=\emptyset$ and $I \cup J=\{1, \ldots, k\}$. We obtain

$$
\begin{aligned}
\partial_{a_{1}} & \ldots \partial_{a_{k}} H\left(x_{i} C f_{m, p}\right) \\
& =\sum_{I, J} \partial_{a_{I}}\left(x_{i}\right) \partial_{a_{J}}\left(f_{m, p}\right)-\left(2 p / \lambda_{2 p}\right) \sum_{I, J} \partial_{a_{I}}\left(|x|^{2}\right) \partial_{a_{J}}\left(\partial f_{m, p} / \partial x_{i}\right) .
\end{aligned}
$$

We write this as $\sum_{\alpha=1}^{5} A_{\alpha}^{i}$, where

$$
\begin{aligned}
A_{1}^{i} & =x_{i} \partial_{a_{1}} \ldots \partial_{a_{k}} f_{m, p}, \\
A_{2}^{i} & =\sum_{l=1}^{k} a_{l}^{i} \partial_{a_{\hat{\imath}}} f_{m, p}, \\
A_{3}^{i} & =-\left(4 p / \lambda_{2 p}\right) \sum_{l=1}^{k}\left\langle a_{l}, x\right\rangle \partial\left(\partial_{a_{\hat{\imath}}} f_{m, p}\right) / \partial x_{i}, \\
A_{4}^{i} & =-\left(2 p / \lambda_{2 p}\right) \sum_{l, l^{\prime}=1}^{k}\left\langle a_{l}, a_{l^{\prime}}\right\rangle \partial\left(\partial_{a_{\hat{l}}{ }^{\prime}} f_{m, p}\right) / \partial x_{i}, \\
A_{5}^{i} & =-\left(2 p / \lambda_{2 p}\right)|x|^{2} \partial\left(\partial_{a_{1}} \ldots \partial_{a_{k}} f_{m, p}\right) / \partial x_{i},
\end{aligned}
$$


where $a_{l}^{i}$ is the $i$-th coordinate of $a_{l}$;

$$
\partial_{a_{\hat{\imath}}}=\partial_{a_{1}} \ldots \widehat{\partial_{a_{l}}} \ldots \partial_{a_{k}}
$$

and

$$
\partial_{\widehat{a l l}^{\prime}}=\partial_{a_{1}} \ldots \widehat{\partial_{a_{l}}} \ldots \widehat{\partial_{a_{l^{\prime}}}} \ldots \partial_{a_{k}}
$$

and ${ }^{\wedge}$ means that the corresponding factor is absent. Similarly, we write

$$
\partial_{a_{k+1}} \ldots \partial_{a_{2 k}} H\left(x_{i} f_{m, p}\right)
$$

as $\sum_{\beta=1}^{5} B_{\beta}^{i}$, where the formulas for $B$ 's are obtained from those of $A$ 's by replacing $C f_{m, p}$ by $f_{m, p}$ and moving up the value of the vectorial index $l$ to $l+k$. Summarizing

$$
\Psi_{m, p+1}^{k}\left(\Phi_{m, p}^{+}(C)\right)\left(a_{1}, \ldots, a_{2 k}\right)=\left(\lambda_{2 p} /\left(2 \lambda_{p}\right)\right) \sum_{i=0}^{m} \sum_{\alpha, \beta=1}^{5}\left\langle A_{\alpha}^{i}, B_{\beta}^{i}\right\rangle .
$$

Using (29) of the Inductive Lemma and its Laplacian

$$
\sum_{i=0}^{m}\left\langle\partial\left(\partial_{a_{I}} C f_{m, p}\right) / \partial x_{i}, \partial\left(\partial_{a_{J}} f_{p}\right) / \partial x_{i}\right\rangle=0, \quad|I|+|J| \leq 2 k-1
$$

we see that all expressions $\sum_{i=0}^{m}\left\langle A_{\alpha}^{i}, B_{\beta}^{i}\right\rangle$ are zero with the exception of the following:

$$
\begin{aligned}
\sum_{i=0}^{m}\left\langle A_{1}^{i}, B_{1}^{i}\right\rangle & =\Psi_{m, p}^{k}(C)\left(a_{1}, \ldots, a_{2 k}\right)|x|^{2} \\
\sum_{i=0}^{m}\left\langle A_{1}^{i}, B_{5}^{i}\right\rangle & =\sum_{i=0}^{m}\left\langle A_{5}^{i}, B_{1}^{i}\right\rangle \\
& =-\left(2 p / \lambda_{2 p}\right)\left\langle\partial_{a_{1}} \ldots \partial_{a_{2 k}} C f_{m, p}, \sum_{i=0}^{m} x_{i} \partial\left(\partial_{a_{k+1}} \ldots \partial_{a_{2 k}} f_{m, p}\right) / \partial x_{i}\right\rangle|x|^{2} \\
& =-\left(2 p(p-k) / \lambda_{2 p}\right)\left\langle\partial_{a_{1}} \ldots \partial_{a_{k}} C f_{m, p}, \partial_{a_{k+1}} \ldots \partial_{a_{2 k}} f_{m, p}\right\rangle|x|^{2} \\
& =-\left(2 p(p-k) / \lambda_{2 p}\right) \Psi_{m, p}^{k}(C)\left(a_{1}, \ldots, a_{2 k}\right)|x|^{2} ; \\
\sum_{i=0}^{m}\left\langle A_{2}^{i}, B_{5}^{i}\right\rangle & =\sum_{i=0}^{m}\left\langle A_{5}^{i}, B_{2}^{i}\right\rangle \\
& =-\left(2 p / \lambda_{2 p}\right) \sum_{l=1}^{k}\left\langle\partial_{a_{\hat{\imath}}} C f_{m, p}, \partial_{a_{l}} \partial_{a_{k+1}} \ldots \partial_{a_{2 k}} f_{m, p}\right\rangle|x|^{2} \\
& =\left(2 p k / \lambda_{2 p}\right) \Psi_{m, p}^{k}(C)\left(a_{1}, \ldots, a_{2 k}\right)|x|^{2} ; \\
\sum_{i=0}^{m}\left\langle A_{5}^{i}, B_{5}^{i}\right\rangle & =2 p^{2} / \lambda_{2 p}^{2} \triangle\left(\Psi_{m, p}^{k}(C)\left(a_{1}, \ldots, a_{2 k}\right)\right)|x|^{4} .
\end{aligned}
$$

Putting these together, we obtain (46). The proof of (47) is analogous and technically much simpler since it does not require differentiation of the harmonic projection formula.

Corollary 1. Let $k \leq l \leq p-k$. Then $\Psi_{m, p}^{k} \mid V_{m+1}^{(2 l, 2 k, 0, \ldots, 0)} \neq 0$ iff $\Psi_{m, q}^{k} \mid V_{m+1}^{(2 l, 2 k, 0, \ldots, 0)}$ $\neq 0$ for (some or) all $q \geq p$. 
Proof. We may assume that $q=p+1$. Let $\Psi_{m, p+1}^{k} \mid V_{m+1}^{(2 l, 2 k, 0, \ldots, 0)}=0$. By (47), we have $\Psi_{m, p}^{k} \circ \Phi_{m, p+1}^{-} \mid V_{m+1}^{(2 l, 2 k, 0, \ldots, 0)}=0$. As noted in Section $2, \Phi_{m ; p+1}^{-}$is an isomorphism on $V_{m+1}^{(2 l, 2 k, 0, \ldots, 0)}$ for $k \leq l \leq p-k$ so that $\Psi_{m, p}^{k} \mid V_{m+1}^{(2 l, 2 k, 0, \ldots, 0)}=0$. The converse is analogous in the use of (46).

Corollary 2. For $m \geq 3$ and $p \geq 2 k, \Psi_{m, p}^{k} \mid V_{m+1}^{(2 k, 2 k, 0, \ldots, 0)} \neq 0$.

Proof. Rigidity (Corollary 2 in Section 3 ) says that $\Psi_{m, 2 k}^{k} \mid V_{m+1}^{(2 k, 2 k, 0, \ldots, 0)} \neq 0$. Now apply Corollary 1 above.

We now assume (45), or equivalently:

$$
\Psi_{m, k+l}^{k} \mid V_{m+1}^{(2 l, 2 k, 0, \ldots, 0)} \neq 0, l \geq k+2 .
$$

By Corollary 1 above, $k+l$ can be replaced by $p \geq k+l$ and this is precisely (44).

To close this section, we finally note that (45) is equivalent to the existence of $C \in S^{2}\left(\mathcal{H}_{m}^{p}\right)$ such that

$$
\Psi_{m, p}^{0}(C)=\ldots=\Psi_{m, p}^{k-1}(C)=0
$$

and

$$
H\left(\Psi_{m, p}^{k}(C)\left(a_{1}, \ldots, a_{2 k}\right)\right) \neq 0, \text { for some } a_{1}, \ldots, a_{2 k} \in \mathcal{H}_{m}^{1} .
$$

This is because by taking the harmonic part of the polynomial $\Psi_{m, p}^{k}(C)\left(a_{1}, \ldots, a_{2 k}\right)$, we land in $\mathcal{H}_{m}^{2(p-k)}\left(\otimes \mathcal{H}_{m}^{2 k}\right)$ of (42) that contains the component $V_{m+1}^{(2(p-k), 2 k, 0, \ldots, 0)}$ in (45) corresponding to the southeast vertex of $\triangle_{k}^{p}$.

\section{The Source Dimension Raising Operator}

In what follows we give a brief account on some facts from the theory of spherical harmonics; for details, cf. [10]. We will consider $p$-homogeneous polynomials $\xi \in \mathcal{P}_{m+2}^{p}$ in the variables $x=\left(x_{0}, \ldots, x_{m}\right) \in \mathbf{R}^{m+1}$ and $x_{m+1} \in \mathbf{R}$ and write $\xi\left(x, x_{m+1}\right)$ when display of the arguments is necessary.

Any $h \in \mathcal{H}_{m+1}^{p}$ has a unique decomposition

$$
h\left(x, x_{m+1}\right)=\sum_{q=0}^{p} H\left(x_{m+1}^{p-q} h_{q}(x)\right), \quad h_{q} \in \mathcal{H}_{m}^{q}, q=0, \ldots, p .
$$

For fixed $q=0, \ldots, p$, we have

$$
H\left(x_{m+1}^{p-q} h_{q}(x)\right)=\frac{(p-q) ! \Gamma(m / 2+q)}{2^{p-q} \Gamma(m / 2+p)}|x|^{p-q} C_{p-q}^{m / 2+q}\left(\frac{x_{m+1}}{|x|}\right) h_{q}(x),
$$

where $\Gamma$ is the Gamma function and $C_{d}^{a}$ is the Gegenbauer polynomial defined by

$$
\begin{aligned}
C_{d}^{a}(t)=\frac{2^{d} \Gamma(a+d)}{d ! \Gamma(a)}[ & t^{d}-\frac{d(d-1)}{2^{2}(a+d-1)} t^{d-2} \\
& \left.+\frac{d(d-1)(d-2)(d-3)}{2^{4} \cdot 1 \cdot 2(a+d-1)(a+d-2)} t^{d-4}-\ldots\right]
\end{aligned}
$$

The factor

$$
|x|^{p-q} C_{p-q}^{m / 2+q}\left(\frac{x_{m+1}}{|x|}\right)
$$


in (51) is a polynomial in $x_{m+1}$ and $|x|^{2}$. For fixed $a$, the normalized Gegenbauer polynomials

$$
2^{a-1} \Gamma(d)\left(\frac{2(a+d) d !}{\pi \Gamma(2 a+d)}\right)^{2} C_{d}^{a}(t), \quad d=0,1, \ldots,
$$

form an orthonormal system on $[-1,1]$ relative to the weight $\left(1-t^{2}\right)^{a-1 / 2}$. Equivalently, we have

$$
\int_{-1}^{1} C_{d}^{a}(t) C_{d^{\prime}}^{a}(t)\left(1-t^{2}\right)^{a-1 / 2} d t=0, \quad d \neq d^{\prime}
$$

and

$$
\int_{-1}^{1} C_{d}^{a}(t)^{2}\left(1-t^{2}\right)^{a-1 / 2} d t=\frac{\pi \Gamma(2 a+d)}{2^{2 a-1} d !(a+d) \Gamma(a)^{2}}
$$

For the exact normalizing constants in the source dimension raising operator, we need an elementary integral formula that allows us to compare integrals on $S^{m+1} \subset$ $\mathbf{R}^{m+2}$ with those on the equatorial hypersphere $S^{m} \subset \mathbf{R}^{m+1}$ (given by $x_{m+1}=0$ ). It is actually the change of variable formula under the transformation

$$
\gamma:[0, \pi] \times \mathbf{R}^{m+1} \rightarrow \mathbf{R}^{m+2}
$$

defined by

$$
\gamma(\phi, x)=(\sin \phi \cdot x, \cos \phi \cdot|x|), \quad x=\left(x_{0}, \ldots, x_{m}\right) \in \mathbf{R}^{m+1}, \quad \phi \in[0, \pi] .
$$

Restricting to the unit spheres, $\gamma$ projects the cylinder $[0, \pi] \times S^{m}$ to $S^{m+1}$ with 0 and $\pi$ corresponding to the north and south poles. The determinant of the Jacobian of $\gamma$ is

$$
(-1)^{m}|x| \sin ^{m} \phi
$$

Now given $\xi \in \mathcal{P}_{m+2}^{r}$ and $\eta$, any function on $[0, \pi]$, the change of variable formula for $\gamma$ gives

$$
\int_{S^{m+1}} \xi(x) \eta\left(x_{m+1}\right) v_{S^{m+1}}=\int_{0}^{\pi} \eta(\cos \phi) \sin ^{m+r} \phi d \phi \cdot \int_{S^{m}} \xi(x) v_{S^{m}} .
$$

Lemma 1. With respect to the decomposition (50), the standard p-eigenmap $f_{m+1, p}: S^{m+1} \rightarrow S_{\mathcal{H}_{m+1}^{p}}$ can be written as

$$
f_{m+1, p}\left(x, x_{m+1}\right)=\left(c_{m, p, q} H\left(x_{m+1}^{p-q} f_{m, q}(x)\right)\right)_{0 \leq q \leq p},
$$

where $x=\left(x_{0}, \ldots, x_{m}\right)$ and

$$
c_{m, p, q}=\left(\begin{array}{l}
p \\
q
\end{array}\right)(m+2 q-1) \frac{(m+q-2) !}{(m+p-1) !} \frac{[m(m+2) \ldots(m+2 p-2)]^{2}}{m(m+1) \ldots(m+p+q-1)} .
$$

Proof. For each $q$, we fix an orthonormal basis $\left\{f_{m, q}^{j_{q}}\right\}_{j_{q}=0}^{N(m, q)} \subset \mathcal{H}_{m}^{q}$. Writing $f_{m, q}$ in terms of these as components, it remains to show that the spherical harmonics in (55) are orthonormal. By the above, up to a constant multiple, the integral

$$
\int_{S^{m+1}} H\left(x_{m+1}^{p-q} f_{m, q}^{j_{q}}(x)\right) H\left(x_{m+1}^{p-q^{\prime}} f_{m, q^{\prime}}^{j_{q^{\prime}}^{\prime}}(x)\right) v_{S^{m+1}}
$$

is equal to

$$
\int_{S^{m+1}} f_{m, q}^{j_{q}}(x) f^{j_{q^{\prime}}^{\prime}}(x) C_{p-q}^{m / 2+q}\left(x_{m+1}\right) C_{p-q^{\prime}}^{m / 2+q^{\prime}}\left(x_{m+1}\right) v_{S^{m+1}} .
$$


By the change of variable formula above, this rewrites as

$$
\int_{0}^{\pi} C_{p-q}^{m / 2+q}(\cos \phi) C_{p-q^{\prime}}^{m / 2+q^{\prime}}(\cos \phi) \sin ^{m+q+q^{\prime}} \phi d \phi \int_{S^{m}} f_{m, q}^{j_{q}}(x) f_{m, q^{\prime}}^{j_{q^{\prime}}^{\prime}}(x) v_{S^{m}}
$$

and orthogonality follows. To finish the proof we work out the normalization constants $c_{m, p, q}$ in (55). To simplify the notation, for fixed $q=0, \ldots, p$, we replace $f_{m, q}^{j}$ by a spherical harmonic $h_{q} \in \mathcal{H}_{m}^{q}$ and assume that

$$
\left|h_{q}\right|^{2}=\frac{N(m, q)+1}{\operatorname{vol}\left(S^{m}\right)} \int_{S^{m}} h_{q}(x)^{2} v_{S^{m}}=1 .
$$

Using (54) again, we compute

$$
\begin{aligned}
\int_{S^{m+1}} H\left(x_{m+1}^{p-q} h_{q}(x)\right)^{2} v_{S^{m+1}} & =\left(\frac{(p-q) ! \Gamma(m / 2+q)}{2^{p-q} \Gamma(m / 2+p)}\right)^{2} \\
& \times \int_{S^{m+1}} C_{p-q}^{m / 2+q}\left(x_{m+1}\right)^{2} h_{q}(x)^{2} v_{S^{m+1}} \\
& =\left(\frac{(p-q) ! \Gamma(m / 2+q)}{2^{p-q} \Gamma(m / 2+p)}\right)^{2} \\
& \times \int_{0}^{\pi} C_{p-q}^{m / 2+q}(\cos \phi)^{2} \sin ^{m+2 q} \phi d \phi \int_{S^{m}} h_{q}(x)^{2} v_{S^{m}} \\
& =\frac{\pi(p-q) ! \Gamma(m+p+q)}{2^{2 p+m-1}(m / 2+p) \Gamma(m / 2+p)^{2}} \int_{S^{m}} h_{q}(x)^{2} v_{S^{m}} .
\end{aligned}
$$

Normalizing in the use of (57), we obtain

$$
\begin{aligned}
\left|H\left(x_{m+1}^{p-q} h_{q}(x)\right)\right|^{2} & =\frac{N(m+1, p)+1}{\operatorname{vol}\left(S^{m+1}\right)} \int_{S^{m+1}} H\left(x_{m+1}^{p-q} h_{q}(x)\right)^{2} v_{S^{m+1}} \\
& =\frac{\pi(p-q) ! \Gamma(m+p+q)}{2^{2 p+m-1}(m / 2+p) \Gamma(m / 2+p)^{2}} \\
& \times \frac{N(m+1, p)+1}{N(m, q)+1} \frac{\operatorname{vol}\left(S^{m}\right)}{\operatorname{vol}\left(S^{m+1}\right)}
\end{aligned}
$$

The value of the ratio of the volumes is known [1]. Instead of recalling however, we note that, for $p=q=0,(58)$ gives just that:

$$
\frac{\operatorname{vol}\left(S^{m}\right)}{\operatorname{vol}\left(S^{m+1}\right)}=\frac{2^{m-1} m / 2 \Gamma(m / 2)^{2}}{\pi \Gamma(m)} .
$$

Substituting this back to (58) and using the dimension formula (11) for the space of spherical harmonics, the value of $c_{m, p, q}$ in (56) follows.

To define the source dimension raising operator, we first split off the component in (55) corresponding to $p=q$. We obtain

$$
f_{m+1, p}\left(x, x_{m+1}\right)=\left(c_{m, p, p} f_{m, p}(x),\left(c_{m, p, q} H\left(x_{m+1}^{p-q} f_{m, p}(x)\right)\right)_{0 \leq q \leq p-1}\right) .
$$

Now let $f: \mathbf{R}^{m+1} \rightarrow V$ be a full harmonic $p$-homogeneous polynomial map. We define

$$
\tilde{f}: \mathbf{R}^{m+2} \rightarrow V \oplus\left(\mathcal{H}_{m+1}^{p} / \mathcal{H}_{m}^{p}\right), \quad \mathcal{H}_{m+1}^{p} / \mathcal{H}_{m}^{p}=\sum_{q=0}^{p-1} \mathcal{H}_{m}^{q}
$$


by

$$
\tilde{f}\left(x, x_{m+1}\right)=\left(c_{m, p, p} f(x),\left(H\left(x_{m+1}^{p-q} f_{m, q}(x)\right)\right)_{0 \leq q \leq p-1}\right) .
$$

We claim that $\tilde{f}$ is spherical if $f$ is. Indeed, $f_{m+1, p}=\tilde{f}_{m, p}$ differs from $\tilde{f}$ by replacing $f_{m, p}$ with $f$ so that

$$
\begin{aligned}
|\tilde{f}|^{2}-\left|f_{m+1, p}\right|^{2} & =|\tilde{f}|^{2}-\left|\tilde{f}_{m, p}\right|^{2} \\
& =c_{m, p, p}^{2}\left(|f|^{2}-\left|f_{m, p}\right|^{2}\right) .
\end{aligned}
$$

Note that, in terms of $\Psi^{0}$, this rewrites as

$$
\Psi^{0}(\tilde{f})=c_{m, p, p}^{2} \Psi^{0}(f)
$$

and the claim follows. If $f: S^{m} \rightarrow S_{V}$ is a $p$-eigenmap, restricting to the unit spheres, we defined the $p$-eigenmap

$$
\tilde{f}: S^{m+1} \rightarrow S_{V \oplus\left(\mathcal{H}_{m+1}^{p} / \mathcal{H}_{m}^{p}\right)} .
$$

We say that $\tilde{f}$ is obtained from $f$ by raising the source dimension. Looking at the components in (59), we see that $\tilde{f}$ is full.

Taking equivalence classes, the source dimension raising operator $f \mapsto \tilde{f}$ gives rise to a map $\Theta_{m, p}: \mathcal{L}_{m}^{p} \rightarrow \mathcal{L}_{m+1}^{p}$. More explicitly, let $f=A \circ f_{m, p}$ with a linear map $A: \mathcal{H}_{m}^{p} \rightarrow V$. Comparing (59) and (60), with obvious notations, we have

$$
\tilde{f}=\left(A \oplus I_{\mathcal{H}_{m+1}^{p}} / \mathcal{H}_{m}^{p}\right) \circ f_{m+1, p}
$$

Thus

$$
\begin{aligned}
\Theta_{m, p}(\langle f\rangle) & =\langle\tilde{f}\rangle=\left(A^{\top} A-I_{\mathcal{H}_{m}^{p}}\right) \oplus 0_{\mathcal{H}_{m+1}^{p} / \mathcal{H}_{m}^{p}} \\
& =\langle f\rangle \oplus 0_{\mathcal{H}_{m+1}^{p}} / \mathcal{H}_{m}^{p} .
\end{aligned}
$$

We obtain that $\Theta_{m, p}$ extends to the $S O(m+1)$-module homomorphism

$$
\Theta_{m, p}: S^{2}\left(\mathcal{H}_{m}^{p}\right) \rightarrow S^{2}\left(\mathcal{H}_{m+1}^{p}\right)
$$

induced by the inclusion $\mathcal{H}_{m}^{p} \subset \mathcal{H}_{m+1}^{p}$.

Remark. Note that, if $f: S^{m} \rightarrow S^{n}$ is a $p$-eigenmap with $n<N(m, p)$ then $\tilde{f}$ has range dimension

$$
n+N(m+1, p)-N(m, p)<N(m+1, p) .
$$

Thus $\Theta_{m, p}$ sends boundary points of $\mathcal{L}_{m}^{p}$ to boundary points of $\mathcal{L}_{m+1}^{p}$

Summarizing, $\Theta_{m, p}$ imbeds $\mathcal{L}_{m}^{p}$ into $\mathcal{L}_{m+1}^{p}$ as a linear slice and the imbedding is equivariant with respect to the inclusion $S O(m+1) \subset S O(m+2)$.

The next lemma follows from the definition of $\Psi_{m, p}^{k}$ in (28).

Lemma 2. Let $C \in S^{2}\left(\mathcal{H}_{m}^{p}\right)$. Then, for $0 \leq k \leq p$, we have

$$
\Psi_{m+1, p}^{k}\left(\Theta_{m, p}(C)\right)\left(\tilde{a}_{1}, \ldots, \tilde{a}_{2 k}\right)=c_{m, p, p}^{2} \Psi_{m, p}^{k}(C)\left(a_{1}, \ldots, a_{2 k}\right),
$$

where $a_{i} \in \mathcal{H}_{m}^{1}, i=0, \ldots, 2 k$, is the orthogonal projection of $\tilde{a}_{i} \in \mathcal{H}_{m+1}^{1}$ to $\mathcal{H}_{m}^{1}$. 
Let $f: S^{m} \rightarrow S_{V}$ be a $p$-eigenmap and assume that it is isotropic of order $k-1$. By $(61), \tilde{f}$ is also isotropic of order $k-1$ that is

$$
\Psi_{m+1, p}^{0}(\langle\tilde{f}\rangle)=\ldots=\Psi_{m+1, p}^{k-1}(\langle\tilde{f}\rangle)=0
$$

and, using (61):

$$
\Psi_{m+1, p}^{k}(\langle\tilde{f}\rangle)\left(\tilde{a}_{1}, \ldots, \tilde{a}_{2 k}\right)=c_{m, p, p}^{2} \Psi_{m, p}^{k}(\langle f\rangle)\left(a_{1}, \ldots, a_{2 k}\right) .
$$

It is a crucial point here that the left hand side is considered as a polynomial in the variables $x=\left(x_{0}, \ldots, x_{m}\right)$ and $x_{m+1}$ that, by looking at the right hand side, actually does not depend on the variable $x_{m+1}$. Taking the harmonic projection $H: \mathcal{P}_{m+2}^{2(p-k)} \rightarrow \mathcal{H}_{m+1}^{2(p-k)}$ of both sides (in all variables) we obtain that

$$
H\left(\Psi_{m+1, p}^{k}(\langle\tilde{f}\rangle)\left(\tilde{a}_{1}, \ldots, \tilde{a}_{2 k}\right)\right) \neq 0
$$

provided that $\Psi_{m, p}^{k}(\langle f\rangle)\left(a_{1}, \ldots, a_{2 k}\right) \neq 0$. This is the consequence of the following elementary:

Lemma 3. Let $\xi \in \mathcal{P}_{m+1}^{2(p-k)}$ be a nonzero polynomial in the variables $x=$ $\left(x_{0}, \ldots, x_{m}\right)$. Then, $\xi$, considered as a polynomial in $\mathcal{P}_{m+2}^{2(p-k)}$ in the variables $x$ and $x_{m+1}$, has nonzero harmonic part $H(\xi) \in \mathcal{H}_{m+1}^{2(p-k)}$.

Proof. If $\xi \in \mathcal{P}_{m+2}^{2(p-k)}$ had vanishing harmonic part then canonical decomposition in $\mathcal{P}_{m+2}^{2(p-k)}$ would imply that $\xi$ is a multiple of $|x|^{2}+x_{m+1}^{2}$. This is impossible since $\xi$ does not depend on the variable $x_{m+1}$.

Thus, finally it remains to exhibit an example of a p-eigenmap $f: S^{m} \rightarrow S_{V}$, $m \geq 3$, that is isotropic of order $k-1, k \geq 1$, and $\Psi_{m, p}^{k}(\langle f\rangle) \neq 0$, since we then raise the source dimension to obtain $\tilde{f}$ with the required nonvanishing property (49) (and land in source dimensions $\geq 4$ ). But the existence of this map follows from rigidity (Corollary 2 of Section 5). In fact, let $f: S^{m} \rightarrow S_{V}$ be a nonstandard $p$ eigenmap such that $\langle f\rangle \in V_{m+1}^{(2 k, 2 k, 0, \ldots, 0)}$. Then, by the main induction hypothesis, $f$ is isotropic of order $k-1$ and, by Corollary 2 , Section $5, \Psi_{m, p}^{k}(\langle f\rangle)\left(a_{1}, \ldots, a_{2 k}\right) \neq 0$ for some $a_{1}, \ldots, a_{2 k} \in \mathcal{H}_{m}^{1}$. Theorem 1 follows.

Remark. It also follows that the source dimension and degree raising operators do not commute. In fact, let $f: S^{m} \rightarrow S_{V}$ be a nonstandard $p$-eigenmap such that $\langle f\rangle \in V_{m+1}^{(2 k, 2 k, 0, \ldots, 0)}$. Then $\left\langle f^{+}\right\rangle \in V_{m+1}^{(2 k, 2 k, 0, \ldots, 0)}$ is still nonzero since degree raising is injective on congruence classes. Since $\Psi_{m, p+1}^{k}\left(\left\langle f^{+}\right\rangle\right)\left(a_{1}, \ldots, a_{2 k}\right) \neq 0$, for some $a_{1}, \ldots, a_{2 k} \in \mathcal{H}_{m}^{1}$ (Theorem 1), by Lemma 3 ,

$$
H\left(\Psi_{m+1, p+1}^{k}\left(\left\langle\left(f^{+}\right)^{\sim}\right\rangle\right)\left(a_{1}, \ldots, a_{2 k}\right)\right) \neq 0,
$$

so that $\left\langle\left(f^{+}\right)^{\sim}\right\rangle$ has nonvanishing component in $V_{m+2}^{(2(p+1-k), 2 k, 0, \ldots, 0)}$. On the other hand, it is impossible for $\left\langle(\tilde{f})^{+}\right\rangle$to reach $V_{m+1}^{(2(p+1-k), 2 k, 0, \ldots, 0)}$ since the top nonvanishing component of $\langle\tilde{f}\rangle$ is in $V_{m+2}^{(2(p-k), 2 k, 0, \ldots, 0)}$ and the desired component $V_{m+2}^{(2(p+1-k), 2 k, 0, \ldots, 0)}$ is in the cokernel of the corresponding degree raising operator $\Phi_{m+1, p}^{+}$. 


\section{The Examples of Mashimo And DeTurck-Ziller}

To prove Theorem 1 for $m=3$ one needs, for each $p \geq 4$ and $k \geq 2,2 k+1 \leq p$, an example of a minimal immersion $f: S^{3} \rightarrow S_{V}$ with homothety $\lambda_{p} / 3$ that is isotropic of order $k-1$ and satisfies the nonvanishing property

$$
H\left(\Psi_{3, p}^{k}(\langle f\rangle)\left(a_{1}, \ldots, a_{2 k}\right)\right) \neq 0,
$$

for some $a_{1}, \ldots, a_{2 k} \in \mathcal{H}_{m}^{1}$. In this section, restricting ourselves to $k=2$, we show that in all the examples of Mashimo [6] and DeTurck-Ziller [4], we have

$$
\Psi_{3, p}^{2}(\langle f\rangle)(a, b, c, d) \equiv 0 \quad\left(\bmod |x|^{2(p-4)}\right),
$$

for all $a, b, c, d \in \mathcal{H}_{3}^{1}$. This means that, taking the canonical decomposition of $\Psi_{3, p}^{2}(\langle f\rangle)(a, b, c, d) \in \mathcal{P}_{4}^{2(p-2)}$, the components of $\Psi_{3, p}^{2}(\langle f\rangle)$ in

$$
V_{4}^{(4,4)}, V_{4}^{(6,4)}, \ldots, V_{4}^{2(p-2)}
$$

all vanish except (possibly) in $V_{4}^{(4,4)}$. The component in $V_{4}^{(4,4)}$ thus vanishes iff $f$ is isotropic of order 2. For example, it follows that the minimum codimension example $f: S^{3} \rightarrow S^{6}$ with homothety $\lambda_{6} / 3$, has vanishing components in $V_{4}^{(6,4)}$ and $V_{4}^{(8,4)}$ but the component in $V_{4}^{(4,4)}$ does not vanish since $f$ is not isotropic of order 2. (If it were, the second osculating bundle would be isomorphic with that of the standard minimal immersion $f_{3,6}$ so that the second osculating space $\mathcal{O}_{f ; o}^{2}$ would be isomorphic, as an $S O(3)$-module, with $\mathcal{H}_{2}^{2}$. This is, however, 5-dimensional so that it does not fit in the 3-dimensional fibre (at $o$ ) of the normal bundle of $f$ ).

To prove (62) we first make a general remark. Let $f: S^{m} \rightarrow S_{V}, m \geq 3$, be a minimal immersion with homothety $\lambda_{p} / m$. We claim that

$$
\Psi_{m, p}^{2}(\langle f\rangle)(a, b, c, d) \equiv\left\langle\partial_{a} \partial_{b} f, \partial_{c} \partial_{d} f\right\rangle \quad\left(\bmod |x|^{2(p-4)}\right) .
$$

This follows by tedious but elementary computation (cf. the analogous statement for $\Psi^{1}$ in [9]) in the use of the formula

$$
\begin{aligned}
\left\langle\beta\left(f_{m, p}\right)(X, Y), \beta\left(f_{m, p}\right)(U, V)\right\rangle= & (c / 2)(\langle X, U\rangle\langle Y, V\rangle+\langle X, V\rangle\langle Y, U\rangle) \\
& -(c / m)\langle X, Y\rangle\langle U, V\rangle,
\end{aligned}
$$

where the value of the constant

$$
c^{2}=\frac{1-m / \lambda_{p}}{1 / 2-1 / m}
$$

follows from the equation of Gauss. (In fact, restricting to the base point $o=$ $(0, \ldots, 0,1) \in S^{m}$, the $S O(m)$-module homomorphism

$$
\beta\left(f_{p}\right)_{o}: S^{2}\left(\mathcal{H}_{m-1}^{1}\right) \rightarrow \mathcal{H}_{m-1}^{2}
$$

is surjective $[5,11]$, so that it must be $B \rightarrow B-(1 / m) \operatorname{trace} B, B \in S^{2}\left(\mathcal{H}_{m-1}^{1}\right)$. Now (64) follows by equivariance.)

Let $\mathcal{V}^{p}$ denote the irreducible complex $S U(2)$-module of polynomials in the complex variables $z, w \in \mathbf{C}$ that are homogeneous of degree $p ; \operatorname{dim}_{\mathbf{C}} \mathcal{V}^{p}=p+1$. The module structure

$$
T: S U(2) \rightarrow G L_{\mathbf{C}}\left(\mathcal{V}^{p}\right)
$$

is given by

$$
T_{g} \mu(z, w)=\mu(\bar{\alpha} z-\beta w, \bar{\beta} z+\alpha w), \quad \mu \in \mathcal{V}^{p},
$$


where

$$
g=\left(\begin{array}{rr}
\alpha & \beta \\
-\bar{\beta} & \bar{\alpha}
\end{array}\right) \in S U(2)
$$

$T$ is unitary with respect to the Hermitian scalar product on $\mathcal{V}^{p}$ given by declaring

$$
\left\{\frac{z^{i} w^{p-i}}{\sqrt{i !(p-i) !}}\right\}_{i=0}^{p}
$$

an orthonormal basis. For fixed $\mu \in \mathcal{V}^{p}$, consider the orbit map

$$
f: S^{3} \rightarrow \mathcal{V}^{p}
$$

given by

$$
f(g)=T_{g} \mu, \quad g \in S U(2)
$$

Then $[4,6]$, under suitable conditions imposed on $\mu$, and up to scaling, $f$ gives rise to a minimal immersion $f: S^{3} \rightarrow S_{\mathcal{V}^{p}}$ with homothety $\lambda_{p} / 3$. (Note that, as in [4], one can consider polynomials $\mu$ depending also on $\bar{z}$ and $\bar{w}$. This requires somewhat more computations but (62) remains true.) To show (62), we suppress the vectors $a, b, c, d \in \mathcal{H}_{3}^{p}$ and, by (63), using complex coordinates $r, s \in \mathbf{C}$, rewrite it as

$$
\Psi_{3, p}^{2}(f) \equiv \Re\left\langle\mathcal{D}_{1} \mu(\bar{r} z-s w, \bar{s} z+r w), \mathcal{D}_{2} \mu(\bar{r} z-s w, \bar{s} z+r w)\right\rangle \quad\left(\bmod \rho^{2(p-4)}\right)
$$

where $\rho^{2}=r \bar{r}+s \bar{s}$ and $\mathcal{D}_{1}$ and $\mathcal{D}_{2}$ are second order differential operators with constant coefficients with respect to the variables $r, \bar{r}, s, \bar{s}$. With these notations, we now claim

$$
\Psi_{3, p}^{2}(f) \equiv 0 \quad\left(\bmod \rho^{2(p-4)}\right)
$$

We take, for example

$$
\mathcal{D}_{1}=\frac{\partial^{2}}{\partial r^{2}} \text { and } \mathcal{D}_{2}=\frac{\partial^{2}}{\partial r \partial s}
$$

the other cases can be treated analogously. We have

$$
\Psi_{3, p}^{2}(f) \equiv \Re\left\langle\frac{\partial^{2} \mu}{\partial w^{2}}(\bar{r} z-s w, \bar{s} z+r w) w^{2}, \frac{\partial^{2} \mu}{\partial z \partial w}(\bar{r} z-s w, \bar{s} z+r w)\left(-w^{2}\right)\right\rangle .
$$

Setting

$$
g=\frac{1}{\rho}\left(\begin{array}{rr}
r & s \\
-\bar{s} & \bar{r}
\end{array}\right) \in S U(2)
$$


and using that $T$ is unitary, we compute

$$
\begin{aligned}
& \Psi_{3, p}^{2}(f) \equiv \Re\left\langle\rho^{p-2} \frac{\partial^{2} \mu}{\partial w^{2}}\left(\frac{\bar{r}}{\rho} z-\frac{s}{\rho} w, \frac{\bar{s}}{\rho} z+\frac{r}{\rho} w\right) w^{2},\right. \\
& \left.\rho^{p-2} \frac{\partial^{2} \mu}{\partial z \partial w}\left(\frac{\bar{r}}{\rho} z-\frac{s}{\rho} w, \frac{\bar{s}}{\rho} z+\frac{r}{\rho} w\right)\left(-w^{2}\right)\right\rangle \\
& =\rho^{2(p-2)} \Re\left\langle\left(T_{g} \frac{\partial^{2} \mu}{\partial w^{2}}\right)(z, w),-\left(T_{g} \frac{\partial^{2} \mu}{\partial z \partial w}\right)(z, w) w^{2}\right\rangle \\
& =\rho^{2(p-2)} \Re\left\langle\frac{\partial^{2} \mu}{\partial w^{2}}(z, w)\left(-\frac{\bar{s}}{\rho} z+\frac{\bar{r}}{\rho} w\right)^{2},\right. \\
& \left.-\frac{\partial^{2} \mu}{\partial z \partial w}(z, w)\left(-\frac{\bar{s}}{\rho} z+\frac{\bar{r}}{\rho} w\right)^{2}\right\rangle \\
& \equiv 0\left(\bmod \rho^{2(p-4)}\right) \text {. }
\end{aligned}
$$

Thus (62) follows.

\section{REFERENCES}

1. Berger M., Geometry I-II, Springer, 1987. MR 88a:51001a; MR 88a:51001b

2. Börner H., Representations of Groups, North Holland, Amsterdam, 1963.

3. Calabi E., Minimal immersions of surfaces in euclidean spheres, J. Diff. Geom., 1 (1967) 111-125. MR 38:1616

4. DeTurck D.-Ziller W., Minimal isometric immersions of spherical space forms in spheres, Comment. Math. Helvetici 67 (1992) 428-458. MR 93f:53050

5. DoCarmo M.-Wallach N., Minimal immersions of spheres into spheres, Ann. of Math., 93 (1971) 43-62. MR 43:4048

6. Mashimo K., Minimal immersions of 3-dimensional sphere into spheres, Osaka J. Math., 21 (1984) 721-732. MR 86d:53040

7. Takahashi T., Minimal immersions of Riemannian manifolds, J. Math. Soc. Japan, 18 (1966) 380-385. MR 33:6551

8. Toth G., Harmonic maps and minimal immersions through representation theory, Academic Press, Boston, 1990. MR 91a:58050

9. Toth G., Eigenmaps and the space of minimal immersions between spheres, Indiana Univ. Math. J. (1994) (to appear).

10. Vilenkin N.I., Special Functions and the Theory of Group Representations, AMS Translations of Mathematical Monographs, Vol. 22, 1968. MR 37:5429

11. Wallach N, Minimal immersions of symmetric spaces into spheres, in Symmetric Spaces, Dekker, New York, 1972, 1-40. MR 53:11545

Department of Mathematics, Eastern Illinois University, Charleston, Illinois 61920

E-mail address: cfhvg@ux1.cts.eiu.edu

Department of Mathematics, Rutgers University, Camden, New Jersey 08102

E-mail address: gtoth@crab.rutgers.edu 